\title{
A pentagonal hydrogen-bond network facilitates an exceptional preference for asparagine in the S2 subsite of Stenotrophomonas maltophilia dipeptidyl peptidase 7
}

Akihiro Nakamura

Nagaoka University of Technology

Yoshiyuki Suzuki

National Institute of Technology (KOSEN), Nagaoka College

Yasumitsu Sakamoto

Iwate Medical University

Saori Roppongi

Iwate Medical University

Chisato Kushibiki

Iwate Medical University

Natsuri Yonezawa

Iwate Medical University

Masato Takahashi

Iwate Medical University

Yosuke Shida

Nagaoka University of Technology

Hiroaki Gouda

Showa University

Takamasa Nonaka

Iwate Medical University

Nobutada Tanaka

Kitasato University

Wataru Ogasawara ( $\square$ owataru@vos.nagaokaut.ac.jp )

Nagaoka University of Technology

\section{Research Article}

Keywords: DPP7s, DPP11s, SmDPP7, Stenotrophomonas maltophilia

Posted Date: January 11th, 2021 
DOl: https://doi.org/10.21203/rs.3.rs-142430/v1

License: (c) (1) This work is licensed under a Creative Commons Attribution 4.0 International License. Read Full License

Version of Record: A version of this preprint was published at Scientific Reports on April 12th, 2021. See the published version at https://doi.org/10.1038/s41598-021-86965-x. 


\section{Abstract}

The emergence of drug-resistant bacteria has become a major problem worldwide. Bacterial dipeptidyl peptidases 7 and 11 (DPP7s and DPP11s), belonging to the family-S46 peptidases, are important enzymes for bacterial growth and are not present in mammals. Therefore, specific inhibitors for these peptidases are promising as potential antibiotics. While the molecular mechanisms underlining strict substrate specificity at the S1 subsite of S46 peptidases have been well studied, those of relatively broad substrate preference at the S2 subsite of these peptidases are unknown. In this study, we performed structural and biochemical analyses on DPP7 from Stenotrophomonas maltophilia (SmDPP7). SmDPP7 showed substrate preference for hydrophobic amino acids at the S2 subsite in general, but as an exception, also for asparagine, a hydrophilic amino acid. Structural analyses of SmDPP7 revealed that this exceptional preference to asparagine is caused by a hydrogen bonding network at the bottom of the S2 subsite. The residues in the S2 subsite are well conserved among S46 peptidases as compared with those in the $\mathrm{S} 1$ subsite. We expect that our findings will contribute toward the development of a universal inhibitor of S46 peptidases. (184 words / 200 words)

\section{Introduction}

Antimicrobial resistance is a worldwide problem ${ }^{1}$. As bacteria are able to circumvent the effects of all the currently available antibiotics, there is an urgent need for the development of new antibiotics with novel modes of action. However, the development of new antimicrobial agents is declining worldwide. Among bacterial pathogens, sugar non-fermenting Gram-negative bacteria (NFGNB) are at the center of the antimicrobial resistance epidemic ${ }^{2}$. Stenotrophomonas maltophilia (formerly named Pseudomonas maltophilia or Xanthomonas maltophilia) ${ }^{3}$ is a NFGNB and is a multiple-drug resistant bacterium Iinvolved in Dopportunistic infections in immunocompromised patients ${ }^{4-6}$. This bacterium exhibits antimicrobial resistance against available carbapenem class of broad-spectrum antibiotics as it possesses multidrug-resistance pumps ${ }^{7,8}$, plasmids harbouring antibiotic resistance genes, and various gene transfer mechanisms involved in the acquisition of antimicrobial resistance.

The World Health Organisation (WHO) recently classified S. maltophilia as one of the leading multidrugresistant organisms (MDROs) in hospital settings ${ }^{9}$. A multitude of factors have been associated with acquiring S. maltophilia infections. These include, underlying malignancy (especially hematologic malignancy), organ transplantation, human immunodeficiency virus (HIV) infection, cystic fibrosis, prolonged hospitalization, intensive care unit (ICU) admission, mechanical ventilation, indwelling catheters (vascular, urinary, biliary), and corticosteroid or immunosuppressive therapy, (treatments concurrently given along with antibiotics administration $)^{10}$. Considering the wide range of these risk factors, novel antimicrobial strategies are needed to combat $S$. maltophilia infections, and these antimicrobials must be highly specific to certain bacteria to avoid the emergence of antimicrobial resistant bacteria. 
Family S46 peptidases are serine proteases showing dipeptidyl peptidase activity and are widely distributed in Bacteroidetes and Proteobacteria, but are not found in mammals ${ }^{11,12}$. Since $S 46$ peptidases are important enzymes for the growth of bacteria ${ }^{13,14}$, these peptidases are anticipated as a novel molecular target of antibiotics ${ }^{15}$. However, a clear difference in the substrate specificity at the P1 position of the substrate between the two types of S46 peptidases has been an obstacle when designing a universal inhibitor. S46 peptidases can be divided into two types, dipeptidyl peptidases 7 (DPP7) and dipeptidyl peptidase 11 (DPP11) according to the substrate specificity at the P1 position ( $\mathrm{NH}_{2}-\mathrm{P} 2-\mathrm{P} 1-\mathrm{P} 1$ 'P2'-..., where the P1-P1' bond is the scissile bond $)^{16}$. DPP11s exhibit a strict substrate specificity for acidic residues (Asp/Glu) at the P1 position, whereas DPP7s exhibit a broad substrate specificity for both aliphatic and aromatic residues at the P1 position. The substrate P1 specificity/preference of S46 peptidases could be inferred according to the amino acid at position 673 of PgDPP1 ${ }^{16}$. DPP7s have glycine, and DPP11s have arginine or serine at position 673 of PgDPP11. As DPP7s have glycine at position 673 , the $\mathrm{S} 1$ subsite of DPP7s can form a pocket deep enough to accommodate bulky hydrophobic residues. On the other hand, DPP11s exhibit specificity for Asp/Glu by the electrostatic interaction between the side chain of Arg673 and the carboxy group of the P1-side chain of Asp/Glu ${ }^{17}$. Because antibiotics targeting S46 peptidases need to inhibit both DPP7 and DPP11, this specificity difference between DPP7 and DPP11 at the P1 position can be a potential obstacle when designing a universal inhibitor of S46 peptidases.

Crystal structure analyses have unravelled the substrate recognition mechanisms of S46 peptidase at the P1 position. S46 peptidases have two subunits forming a homodimer, with each subunit consisting of approximately 770 amino acid residues. The first three-dimensional structure of an S46 peptidase was determined for dipeptidyl aminopeptidase BII (DAP BII), a DPP7-type enzyme, from Pseudoxanthomonas mexicana WO24 ${ }^{18}$. The study revealed that a protomer of DAP Bll contains a peptidase domain, including a double $\beta$-barrel fold that is characteristic of the chymotrypsin superfamily, as well as an unusual ahelical domain that regulates the exopeptidase activity of DAP BII. Subsequently, crystal structures of dipeptidyl peptidase 11 from Porphyromonas gingivalis (PgDPP11) were identified ${ }^{17,19}$. Crystal structure analyses and biochemical studies of PgDPP $11^{16,20}$ have revealed that Arg673 in PgDPP11 is responsible for the strict Asp/Glu specificity of PgDPP11 at the P1 position of the substrate peptide. Thus, the overall structure, the molecular basis of the exopeptidase activity, the catalytic mechanism, and the strict P1residue recognition mechanisms of S46 peptidases have been unravelled by the crystal structure analyses of DAP BII and PgDPP11 ${ }^{17-19}$. However, little is known about the mechanism of P2-residue recognition.

Biochemical studies of DPP7 from P. gingivalis (PgDPP7) and PgDPP11 suggested that the side chain of Phe664 in PgDPP7 and of Phe671 in PgDPP11 are involved in the recognition of the hydrophobic P2 residue of the substrate peptide. This hydrophobic specificity at the $\mathrm{P} 2$ position is conserved between DPP7 and DPP11 21 . The conservation of substrate specificity has provided access to design the universal inhibitor of S46 peptidases, however, determinants for the P2-residue specificity/preference of S46 peptidases at the atomic level remain to be fully elucidated. Therefore, understanding the substrate 
recognition mechanism at the $\mathrm{S} 2$ subsite could provide more insight for the development of a universal inhibitor of S46 peptidases. It should be noted that in mammals, a peptidase designated DPP7 is one of

the two members of the $S 28$ peptidase family ${ }^{22,23}$ and is distinct from bacterial DPP7s that belong to the S46 peptidase family. The S28 peptidases contain two major domains: a peptidase domain, including an a/ $\beta$-hydrolase fold, and an a-helical bundle.

In this study, we evaluated the P2-residue preference of DPP7 from S. maltophilia (SmDPP7) and determined the crystal structures of SmDPP7 in complexes with four kinds of dipeptides (Val-Tyr, Phe-Tyr, Tyr-Tyr, and Asn-Tyr) at resolutions of 2.03 to $1.88 \AA ̊$. Biochemical studies showed a preference for bulky hydrophobic residues $(F, L>Y, M>>V)$ at the $S 2$ subsite of SmDPP7, consistent with a previous report for other DPPs ${ }^{21}$. However, a notable exception newly uncovered in this study was a strong preference for the asparagine residue at the $\mathrm{S} 2$ subsite. Crystal structure analysis of the Asn-Tyr complex revealed that the exceptionally strong preference for asparagine residue is facilitated by a water-mediated hydrogen bond network in the $\mathrm{S} 2$ subsite. The binding mode of the asparagine residue at the $\mathrm{S} 2$ subsite revealed by the crystal structure analysis was consistent with the result of isothermal titration calorimetry (ITC) analysis for the binding of Asn-Tyr to SmDPP7, which showed that the binding interaction is clearly enthalpydriven. An extensive amino acid sequence comparison revealed that residues in the S2 subsite of S46 peptidases are better conserved than those in the $\mathbf{S 1}$ subsite. Indeed, competitive inhibition assay using dipeptides against other $\mathrm{S} 46$ peptidases from pathogenic bacteria showed a conserved preference for hydrophobic residues and asparagine at the $\mathrm{S} 2$ subsite. These observations provide novel insights into the design of a universal inhibitor of S46 peptidases.

\section{Results}

\section{P2 position preferences of SmDPP7}

[First, we evaluated the P1 preferences of SmDPP7 and several other DPPs by using synthetic substrates (Figure S1). SmDPP7 showed clear preferences for hydrophobic and basic amino acids at the P1 position. This result was in agreement with previous reports, in which PmDAP BII and PgDPP7 showed substrate $\mathrm{P} 1$ preferences against hydrophobic and basic amino acids ${ }^{11,24}$. In order to quantitatively estimate the substrate preference at the $\mathrm{P} 2$ position, inhibitory effects of the dipeptides Xaa-Tyr against the hydrolytic activity of wild-type SmDPP7 were evaluated on a synthetic substrate, tyrosyl-L-tyrosine 4methylcoumaryl-7-amide (Tyr-Tyr-MCA). Here, Xaa indicates any amino acid except for Cys, because CysTyr was not included in the commercially available dipeptide library (AnaSpec, Fremont CA, USA) and the synthetic service (Eurofins genomics, JN) that we used. The hydrolytic activity of SmDPP7 was markedly inhibited by Phe-Tyr, Leu-Tyr, Tyr-Tyr, Asn-Tyr, and Met-Tyr, the top five of the 19 dipeptides shown in descending order (Fig. 1). The inhibitory constants $\left(K_{\mathrm{i}}\right)$ of Phe-Tyr, Leu-Tyr, Tyr-Tyr, and Met-Tyr were 1.27, $2.39,7.66$ and $11.2 \mu \mathrm{M}$, respectively, and these were clearly aligned with the hydrophobicity indexes (H.I.) of P2 amino acids ${ }^{25,26}$ (Table 1). The correlation coefficient between the residual activity of SmDPP7 and H.I. of the P2 amino acid of dipeptides was -0.748 when the outlier dipeptides Asn-Tyr and Trp-Tyr (red 
and blue, respectively, in Fig. 2a) were excluded, whereas the value was - 0.491 when all the dipeptides were considered. The negative correlations between the residual activity and the H.I. of the P2 amino acid of dipeptides indicated that SmDPP7 prefers the hydrophobic amino acids at the P2 position. Interestingly, Asn-Tyr (H.I. of Asn: - 28) showed an exceptional inhibitory effect with $K_{\mathrm{i}}$ value of 7.80 against SmDPP7. In order to evaluate the characteristics of the Asn-Tyr dipeptide as a substrate of SmDPP7, the kinetic parameters of SmDPP7 for Asn-Tyr-MCA were determined (Table 2). The specificity constant $\left(k_{\text {cat }} / K_{\mathrm{m}}\right)$ for Asn-Tyr-MCA of SmDPP7 was $71.5 \mathrm{sec}^{-1} \mathrm{mM}^{-1}$, which was 1.5 times higher than that for Tyr-Tyr-MCA. This result indicated that SmDPP7 prefers a substrate with a P2-Asn residue (AsnTyr-MCA), as is the case with a product dipeptide (Asn-Tyr). We further examined the binding of amino acids at the S2 subsite of SmDPP7 by structural and site-directed mutagenesis studies, as described below. 
Table 1

Inhibition constants $\left(K_{\mathrm{i}}\right)$ of Xaa-Tyr / Xaa-Asp dipeptides against the hydrolytic activities on synthetic substrates of S46 peptidases.

\begin{tabular}{|c|c|c|c|c|c|}
\hline \multirow[t]{2}{*}{ P2 amino acid (Xaa) } & \multirow[t]{2}{*}{ H.I. of P2 aa } & \multicolumn{4}{|l|}{$K_{\mathrm{i}}(\mu \mathrm{M})$} \\
\hline & & SmDPP7 & PgDPP7 & SmDPP11 & PgDPP11 \\
\hline Asp & -55 & - & - & - & - \\
\hline Pro & $-46^{*}$ & - & - & - & - \\
\hline Glu & -31 & - & - & - & - \\
\hline Asn & -28 & $7.80 \pm 0.42$ & $210 \pm 18$ & $61.9 \pm 6.6$ & $4.06 \pm 0.16$ \\
\hline Lys & -23 & - & - & - & - \\
\hline Arg & -14 & - & - & $23.2 \pm 1.4$ & - \\
\hline Gln & -10 & - & - & - & - \\
\hline Ser & -5 & - & - & - & - \\
\hline Gly & 0 & - & - & - & - \\
\hline $\mathrm{His}$ & 8 & - & - & - & - \\
\hline Thr & 13 & - & - & - & - \\
\hline Ala & 41 & - & - & - & - \\
\hline Tyr & 63 & $7.66 \pm 1.27$ & - & $5.66 \pm 0.23$ & - \\
\hline Met & 74 & $11.2 \pm 0.4$ & - & - & - \\
\hline Val & 76 & $55.2 \pm 0.5$ & - & - & - \\
\hline Trp & 97 & - & $30.6 \pm 0.9$ & $6.27 \pm 0.18$ & $1.36 \pm 0.06$ \\
\hline Leu & 97 & $2.39 \pm 0.08$ & $61.0 \pm 1.2$ & $5.68 \pm 0.07$ & $3.35 \pm 0.23$ \\
\hline Ile & 99 & - & - & - & - \\
\hline Phe & 100 & $1.27 \pm 0.06$ & $150 \pm 2$ & $7.01 \pm 0.27$ & $5.11 \pm 0.06$ \\
\hline $\begin{array}{l}\text { Xaa-Tyr and Xaa-Asp } \\
\text { and Leu-Asp-MCA we } \\
\text { indexes (H.I.) of the a } \\
\text { *The hydrophobicity } \\
\text { 2.0. "-" means not det } \\
\text { three independent exp }\end{array}$ & $\begin{array}{l}\text { used as subs } \\
\text { sed as substrat } \\
0 \text { acids were ac } \\
x \text { of Proline is n } \\
\text { ined due to low } \\
\text { hents. }\end{array}$ & $\begin{array}{l}\text { tes for DPP7s } \\
\text { for DPP7s ar } \\
\text { oted from Ser } \\
\text { malized from } \\
\text { hibitory activ }\end{array}$ & $\begin{array}{l}\text { nd DPP11s } \\
\text { DPP11s, re } \\
\text { a et al., } 199 \\
\text { ereda et al., } \\
\text { Standard }\end{array}$ & $\begin{array}{l}\text { spectively, an } \\
\text { ectively. The } h \\
\text { ind Mohera e } \\
94 \text { under the } \\
\text { iations were }\end{array}$ & $\begin{array}{l}\text { Tyr-Tyr-MCA } \\
\text { rophobicity } \\
\text { l. } 1995^{25,26} \\
\text { ndition at pH } \\
\text { tained from }\end{array}$ \\
\hline
\end{tabular}


Table 2

Kinetic parameters of wild-type and mutant SmDPP7s toward each synthetic substrate

\begin{tabular}{|c|c|c|c|c|}
\hline Variation & Substrate & $K_{\mathrm{m}}(\mu \mathrm{M})$ & $k_{c a t}\left(\sec ^{-1}\right)$ & $k_{c a t} / K_{\mathrm{m}}\left(\sec ^{-1} \mathrm{mM}^{-1}\right)$ \\
\hline \multirow[t]{3}{*}{ Wild type } & Gly-Tyr-MCA & $183 \pm 4$ & $0.702 \pm 0.022$ & $3.85 \pm 0.03$ \\
\hline & Tyr-Tyr-MCA & $81.7 \pm 3.4$ & $3.83 \pm 0.09$ & $46.9 \pm 1.2$ \\
\hline & Asn-Tyr-MCA & $51.3 \pm 1.6$ & $3.67 \pm 0.12$ & $71.5 \pm 0.3$ \\
\hline \multirow[t]{3}{*}{ K206A } & Gly-Tyr-MCA & $201 \pm 10$ & $0.751 \pm 0.015$ & $3.73 \pm 0.13$ \\
\hline & Tyr-Tyr-MCA & $55.8 \pm 1.5$ & $3.52 \pm 0.04$ & $63.2 \pm 1.5$ \\
\hline & Asn-Tyr-MCA & $54.1 \pm 2.1$ & $3.47 \pm 0.02$ & $64.4 \pm 2.6$ \\
\hline \multirow[t]{3}{*}{ R218A } & Gly-Tyr-MCA & - & - & - \\
\hline & Tyr-Tyr-MCA & - & - & - \\
\hline & Asn-Tyr-MCA & - & - & - \\
\hline \multirow[t]{3}{*}{ R218Q } & Gly-Tyr-MCA & $717 \pm 63$ & $0.0365 \pm 0.0018$ & $0.0510 \pm 0.0020$ \\
\hline & Tyr-Tyr-MCA & $275 \pm 11$ & $0.0403 \pm 0.0004$ & $0.146 \pm 0.005$ \\
\hline & Asn-Tyr-MCA & $390 \pm 14$ & $0.198 \pm 0.006$ & $0.509 \pm 0.020$ \\
\hline \multirow[t]{3}{*}{ R218K } & Gly-Tyr-MCA & $586 \pm 40$ & $0.0238 \pm 0.0011$ & $0.0407 \pm 0.0017$ \\
\hline & Tyr-Tyr-MCA & $251 \pm 6$ & $0.240 \pm 0.003$ & $0.955 \pm 0.012$ \\
\hline & Asn-Tyr-MCA & $277 \pm 6$ & $0.183 \pm 0.008$ & $0.660 \pm 0.017$ \\
\hline \multirow[t]{3}{*}{ T220A } & Gly-Tyr-MCA & $215 \pm 10$ & $0.454 \pm 0.010$ & $2.12 \pm 0.06$ \\
\hline & Tyr-Tyr-MCA & $46.4 \pm 2.4$ & $1.82 \pm 0.07$ & $39.2 \pm 0.7$ \\
\hline & Asn-Tyr-MCA & $66.6 \pm 1.5$ & $2.60 \pm 0.04$ & $39.0 \pm 0.3$ \\
\hline \multirow[t]{3}{*}{ F671A } & Gly-Tyr-MCA & $688 \pm 38$ & $0.191 \pm 0.009$ & $0.278 \pm 0.003$ \\
\hline & Tyr-Tyr-MCA & $463 \pm 88$ & $1.54 \pm 0.18$ & $3.36 \pm 0.23$ \\
\hline & Asn-Tyr-MCA & $17.4 \pm 1.4$ & $0.230 \pm 0.012$ & $13.7 \pm 0.4$ \\
\hline
\end{tabular}

\section{The overall structure of SmDPP7}

Crystal structures of SmDPP7 in complexes with dipeptides Val-Tyr, Phe-Tyr, Tyr-Tyr, and Asn-Tyr were determined at resolutions of 2.03 to $1.88 \AA$ (Tables S1 and S2). Representative electron density maps of the bound dipeptides are shown in Figure S2. The SmDPP7 enzyme forms a homodimer, with each 
subunit consisting of 697 residues (Ala23-Ala719) and a molecular weight of approximately $155 \mathrm{kDa}$ (Fig. 3). A dimer of SmDPP7 is situated in the asymmetric unit (Fig. 3a). Two protomers of SmDPP7 are related by a non-crystallographic two-fold axis and form a dimer. Dimerisations have also been observed in the crystal structures of other S46 peptidases, PmDAP BII and PgDPP $11^{17-19}$. The protruding $\beta$-hairpin dimerisation interface reported in the above S46 peptidases is also observed for SmDPP7. Each subunit contains a catalytic double $\beta$-barrel domain harbouring the Asp-His-Ser catalytic triad (Fig. 3b, top domain) and an a-helical domain that caps the active site (Fig. 3b, bottom domain). The assignment of the secondary structural elements is provided in Figure S3.

The catalytic domain includes residues $23-274$ and 572-720 and contains a double $\beta$-barrel structure. The $\beta$-barrel structure is a characteristic of the chymotrypsin superfamily. The catalytic domain of SmDPP7 can be superimposed on those of PmDAP Bll and PgDPP11 (Fig. 4a). The serine peptidase catalytic triads, His84, Asp222 and Ser655 in SmDPP7 and His86, Asp224 and Ser657 in PmDAP BII, are almost completely superimposable, with a root mean square ( $\mathrm{rms}$ ) deviation between the two structures of $0.355 \AA$ for 397 structurally equivalent $\mathrm{Ca}$ atoms that had $84.0 \%$ sequence identity for that region. Similarly, the rms deviation between the catalytic domains of SmDPP7 and PgDPP1 1 is $1.01 \AA$ for 308 structurally equivalent $\mathrm{Ca}$ atoms that had $41.9 \%$ sequence identity for that region. Although the sequence identity of the catalytic domains between SmDPP7 and PgDPP11 is low, the catalytic triad residues (His, Asp, and Ser) of both enzymes can be superposed (Fig. 4a, right-side).

The a-helical domain is inserted between strands $\beta 9$ and $\beta 10$ of the catalytic domain and spans residues 275-571 (Figure S3). The domain consists of 15 helices packed into a helical bundle that caps the catalytic triad of the catalytic domain. No structural homologue of this domain is found in the DALI database, except for the a-helical domains of the other structurally characterized S46 peptidases, PmDAP BII and PgDPP11 17,18. Thus, the a-helical domain is absolutely restricted to S46 peptidases. The a-helical domain of SmDPP7 can be superimposed on that of PmDAP BII and PgDPP11 (Fig. 4b). The rms deviation between the a-helical domains of SmDPP7 and PmDAP BII is $0.636 \AA$ for 279 structurally equivalent $\mathrm{Ca}$ atoms across all 297 pairs, which had $68.5 \%$ sequence identity for that region. The rms deviation between the a-helical domains of SmDPP7 and PgDPP11 is $1.38 \AA$ for 66 structurally equivalent $\mathrm{Ca}$ atoms across all 285 pairs, which had $19.1 \%$ sequence identity for that region. In particular, the a-helical domain of PgDPP11 exhibits different inter-domain orientation (Fig. 4b, blue) as compared with those of SmDPP7 and PmDAP BII (Fig. 4b, right). Thus, we found that the structure of the a-helical domain of SmDPP7 is similar to that of PmDAP Bll but is difficult to superpose onto that of PgDPP11.

\section{Dipeptide complexes}

The four dipeptide complexes clearly show the molecular basis for substrate recognition mechanism of SmDPP7. For simplicity, the following description refers primarily to subunit A of the 1.88 -Å-resolution structure of the Tyr-Tyr complex of SmDPP7. The bound dipeptide was found in the active site cleft of the catalytic domain (Fig. 3b) and was covered by the a-helical domain. SmDPP7 hydrolyses peptides from the $\mathrm{N}$-terminus of oligopeptides, cleaving the dipeptide units $\left(\mathrm{NH}_{2}-\mathrm{P} 2-\mathrm{P} 1-\mathrm{COOH}\right)$ when the second P1 
residue is a hydrophobic amino acid. To act as a dipeptidyl aminopeptidase, SmDPP7 must fix the Nterminus of the substrate peptide in position. The $\mathrm{N}$-terminal amino group recognition residues of SmDPP7 are Asn213, Trp214, and Asp672 from the catalytic domain and Asn328 from the a-helical domain (Fig. 3c). For PmDAP BII, a large-scale conformational change, from open to closed, was observed upon peptide binding ${ }^{18}$. The distance between the $\mathrm{Ca}$ atoms of Asn330 belonging to the ahelical domain and catalytic Ser657 belonging to the catalytic domain of PmDAP Bll observed in the peptide-free, open conformation (PDB ID: 3WOK, Fig. 4C, grey) was $16.6 \AA$, whereas that observed in the dipeptide-bound, closed conformation (PDB ID: 3WOL, Fig. 4c, yellow) was $12.8 \AA$. The corresponding distance between the Ca atoms of Asn328 and Ser655 of SmDPP7 observed in the current dipeptidebound conformation was $12.7 \AA$ (Fig. 4c, pink). This result suggests that the present structure of SmDPP7 corresponds to the closed conformation of PmDAP BII and that the active site cleft of SmDPP7 is closed upon peptide binding. The catalytic triad of SmDPP7 is composed of His84, Asp222, and Ser655. The hydroxy group of Ser655 is hydrogen bonded to the imidazole group of His84 (OG(Ser655)-NE2(His84): $2.9 \AA$ ). One of the oxygen atoms of the carboxyl group of Asp222 forms a hydrogen-bond with His84 and completes the catalytic triad (ND1(His84)--OD2(Asp222): $2.7 \AA$ ). The oxyanion hole is formed by the backbone amide nitrogen atoms of Ser655 and Gly653, and the backbone NH group of P1 residue of the bound peptide is recognised by a hydrogen bond with the carbonyl oxygen of Phe671 (Fig. 3d).

The 11 subsite of SmDPP7 is observed adjacent to the catalytic Ser655 and the oxyanion hole (Fig. 3e). The S1 subsite consists of Asn335, Asp649, lle650, Thr651, Gly652, Ala670, Gly673, Ser677, Val678, Ser680, Asn681, and Met691. Gly673 in SmDPP7, corresponds to Arg673 in PgDPP11, which is a crucial residue for the P1 residue specificity/preference of S46 peptidases (relatively loose preference of DPP7 and Asp/Glu specificity of DPP11) $16,17,20$, and is located in the wall of the S1 subsite. Thus, the S1 subsite of SmDPP7 is large and deep enough to accommodate any amino acid as the P1 residue of the substrate peptide. The aromatic ring of the bound $\operatorname{Tyr}(\mathrm{P} 1)$ has hydrophobic contacts with the side chains of Trp214 and Ile650. The hydroxyl group of the bound Tyr(P1) points toward the bottom of the S1 subsite. There are four buried water molecules at the bottom of the S1 subsite and they constitute a hydrogen bond network with the main-chain and side-chain atoms in the S1 subsite. The hydroxyl group of the bound $\operatorname{Tyr}(\mathrm{P} 1)$ is involved in the hydrogen bond network of the water molecules (not shown in Fig. 3e).

The S2 subsite of SmDPP7 composed of His84, Gly205(C = 0), Lys206, Phe207(C = 0), Gly208, Gly209, Asp210, Arg218, Thr220, Asp222, and Phe671 is sufficiently wide enough to accommodate a bulky side chain (Fig. 3e). The side chain of bound Tyr(P2) is accommodated in the S2 subsite and has aliphatic contacts with the side chains of His84, Lys206, and Phe671 (Fig. 5a). The hydroxyl group of Tyr(P2) forms hydrogen bonds to the carbonyl oxygens of Phe207 and Gly209, and the side chain of Arg218 via a water molecule. Thus, specific interaction between the $\mathrm{S} 2$ subsite and the side chain of $\operatorname{Tyr}(\mathrm{P} 2)$ is limited, whereas the main-chain atoms of $\operatorname{Tyr}(\mathrm{P} 2)$ are tightly fixed by the side chains of Asn213, Trp214, Asn328, 
and Asp672 as described above. This is consistent with the lack of a strict specificity of SmDPP7 for the $\mathrm{P} 2$ residue.

For the Val-Tyr complex, some differences were observed in the intermolecular interactions around the S2 subsite as compared to those for the Tyr-Tyr complex, while intermolecular interactions between the side chain of $\operatorname{Tyr}(\mathrm{P} 1)$ and the residues in the S1 subsite were almost identical between the Val-Tyr and Tyr-Tyr complexes (not shown in Fig. 5b). The unique features of the Val-Tyr complex are that the bottom space of the S2 subsite is occupied by four water molecules (Fig. 5b) and the side chain of Lys206 is less

ordered. In the Tyr-Tyr complex, one of the four water molecules at the bottom of the S2 subsite in the ValTyr complex is replaced by the hydroxyl group of Tyr(P2) and the side chain of Lys206 is well ordered due to aliphatic contacts with the side chain of $\operatorname{Tyr}(\mathrm{P} 2)$. For the Phe-Tyr complex, intermolecular interactions around the active site are almost conserved as compared with that of the Tyr-Tyr complex. The side chain of Lys206 is well ordered and the five water molecules observed at the bottom of the S2 subsite of the Tyr-Tyr complex are also observed for the Phe-Tyr complex (Fig. 5c). For the Asn-Tyr complex, an extensive hydrogen-bond network was formed at the bottom of the S2 subsite (Fig. 5d). Interestingly, a pentagonal hydrogen-bond network consisting of $\mathrm{HOH} 35, \mathrm{HOH} 63, \mathrm{HOH} 67, \mathrm{HOH} 1112$, and the ND2 atom of P2-Asn was observed (Figure S4). Thus, the exceptional Asn(P2) preference of SmDPP7 could be explained by the water-mediated hydrogen-bond network at the bottom of the S2 subsite of SmDPP7.

\section{Site-directed mutagenesis studies on residues in the S2 subsite of SmDPP7}

To test the roles of the residues located in the S2 subsite for P2 residue recognition by SmDPP7, we replaced the following residues with alanine and analysed the enzymatic activities of the mutant enzymes on synthetic substrates, Gly-Tyr-MCA, Tyr-Tyr-MCA, and Asn-Tyr-MCA (Table 2). The mutated residues and their estimated roles were: Lys206, Arg218, Thr220, and Phe671 for interaction with the P2 side chain. The Arg218 to Ala mutant (R218A) showed complete loss of activity, whereas the F671A mutant resulted in a significant loss of activity (approximately $7-20 \%$ of the $k_{\text {cat }} / K_{\mathrm{m}}$ value of the wildtype enzyme). For the F671A mutant, specificity constant $\left(k_{\text {cat }} / K_{\mathrm{m}}\right)$ values for the Gly-Tyr-MCA, Tyr-TyrMCA, and Asn-Tyr-MCA substrates were significantly decreased. This result indicates that Phe671 in SmDPP7 plays an important role in the accommodation of the substrate peptide into the catalytic domain. The complete loss of the activity of R218A indicates that this residue is important for fixing the carboxyl group of Asp672 in position, which is involved in the recognition of the $\mathrm{N}$-terminus of the substrate peptide. The R218Q and R218K mutants retain slight dipeptidyl peptidase activities. Comparison of $\mathrm{R} 218 \mathrm{Q}$ and $\mathrm{R} 218 \mathrm{~K}$ mutants revealed that $\mathrm{R} 218 \mathrm{~K}$ shows higher activity for all the dipeptidyl substrates examined than R218Q. The length of the Gln side chain of the R218Q mutant appears to be insufficient to fix the side chain of Asp672. The T220A mutant showed moderate loss of activity, whereas the K206A mutant retained activity comparable to that of the wild-type enzyme. For the T220A mutant, $k_{\text {cat }} / K_{\mathrm{m}}$ values for all the substrates examined decreased to approximately $16-45 \%$ of the $k_{\text {cat }} / K_{\mathrm{m}}$ value of the wild-type enzyme, which is attributed to the decreased $k_{\text {cat }}$ value. It is noteworthy that $k_{\text {cat }} / K_{\mathrm{m}}$ values of the K206A mutant for Tyr-Tyr-MCA and Asn-Tyr-MCA were significantly changed from 
that of the wild-type enzyme as compared with that for Gly-Tyr-MCA. Considering that Gly-Tyr-MCA possessed no side chain at the P2 position, the change in $k_{\text {cat }} / K_{\mathrm{m}}$ values for Tyr-Tyr-MCA and Asn-TyrMCA of K206A suggested that the Lys206 residue is involved in an interaction with the P2 side chain.

\section{Thermodynamic characterization of dipeptide bindings in SmDPP7}

Crystal structure analysis of SmDPP7 complexed with Asn-Tyr indicated that a hydrogen bond network consisting of water molecules and the side chain of bound Asn contributes to the exceptional P2-Asn preference in the S2 subsite of SmDPP7, as described above. The thermodynamic parameters of dipeptide bindings in SmDPP7 were determined using isothermal titration calorimetry (ITC) (Figs. 6, S5, and Table S3). Here, we analysed bindings of five dipeptides, Asn-Tyr, Tyr-Tyr, Val-Tyr, Leu-Tyr, and Phe-

Tyr. The bindings with all of the dipeptides examined in SmDPP7 were an enthalpy-driven process with an unfavourable entropic $\square$ contribution. Because S46 peptidases involve conformational changes upon dipeptide binding, which reduce the degrees of freedom of the polypeptide chain (Fig. $3 \mathrm{c})^{18}$, the transition from open to closed state is entropically unfavourable. For Val-Tyr, thermodynamic parameters were not determined due to low-avidity $\left(K_{d}>40 \mu \mathrm{M}\right)$.

\section{P2 position preference of S46 peptidases}

To clarify whether the substrate preferences at the S2 subsite of S46 peptidases are conserved, we analysed P2 residue preference for other S46 peptidases, PgDPP7, SmDPP11 (DPP11 from $S$. maltophilia), and PgDPP11 (Figs. 2b, c, and d). The correlation coefficients between the residual activities of the peptidases and the H.I. of the P2 residue of dipeptides used as competitive inhibitors were -0.642 , - 0.453, and - 0.491, for PgDPP7, SmDPP11, and PgDPP11, respectively. The S46 peptidases showed a preference for hydrophobic amino acids at the P2 position, this tendency was particularly obvious for DPP7s. The preferences of Trp(P2) for SmDPP7, and Met(P2) and Arg(P2) for SmDPP11 were out of trend and exceptional. Interestingly, Asn(P2) preference was conserved among the S46 peptidases analysed. A dipeptide harbouring Asn at the P2 position (Asn-Tyr or Asn-Asp) inhibited PgDPP7, PgDPP11, and SmDPP11 with $K_{\mathrm{i}}$ values of 210, 4.06 and $61.9 \mu \mathrm{M}$, respectively (Table 1). These results indicated that substrate recognition mechanisms at the S2 subsite are partly conserved between DPP7s and DPP11s.

\section{Discussion}

In this study, we evaluated the P2 residue preference of DPP7 from S. maltophilia (SmDPP7), which is distinct from mammalian DPP7s that belong to the $\$ 28$ peptidase family, to better understand determinants for the $\mathrm{P} 2$ residue specificity/preference of $\mathrm{S} 46$ peptidases. We solved the crystal structures of SmDPP7 in the presence of four kinds of dipeptides, Val-Tyr, Phe-Tyr, Tyr-Tyr, and Asn-Tyr. SmDPP7 showed substrate preference for bulky hydrophobic amino acids (except for Trp) and Asn at the P2 position (Figs. 1, 2a, and Table 1). It should be noted that inhibition assays with dipeptides consisting of 
the same P1 amino acids reflect P2 preference of DPPs. SmDPP7 preference for bulky hydrophobic amino acids at the $\mathrm{P} 2$ position is reasonable against the structure of the S2 subsite, which is sufficiently wide enough to accommodate the large side chain (Fig. 3e). Preference for hydrophobic residues at the P2 position by SmDPP7 is in agreement with previous reports ${ }^{21,24}$. Nemoto et al. reported that PgDPP7 and PgDPP11 exhibited a preference for hydrophobic amino acids at the P2 position of various dipeptidyl substrates and that the Phe664 (PgDPP7 numbering) is involved in the recognition of P2 hydrophobic amino acids ${ }^{21}$. In this study, the F671A mutant of SmDPP7, which corresponds to the Phe664 in PgDPP7, showed remarkably decreased specificity constant toward not only hydrophobic dipeptidyl substrates, but also hydrophilic amino acids at the P2 position (Table 2). This indicated that the side chain of Phe671 (numbering in SmDPP7) appears to be important for maintaining the conformation of the S2 subsite rather than for the recognition of $\mathrm{P} 2$ hydrophobic amino acids. However, the specificity for Asn(P2) residue by DPPs has never been reported for bacterial DPPs or mammalian DPPs ${ }^{27}$. To confirm whether the preference for bulky hydrophobic amino acids and Asn at the P2 position was conserved in S46 peptidases, we performed a multiple sequence alignment analysis against 4931 genes coding $\$ 46$ peptidases. We observed that the residues in the S2 subsite are highly conserved among S46 peptidases (Table S4), indicating that it is likely that $\$ 46$ peptidases would share a common substrate preference at the P2 position. Indeed, P2 preference for bulky hydrophobic amino acids and Asn are conserved among S46 peptidases examined in this study (Fig. 2). In contrast, the substrate specificity at the P1 position is distinct between DPP7 and DPP11 in S46 peptidases (Figure S1). SmDPP7 and PgDPP7 showed substrate specificity for hydrophobic and basic amino acids at the P1 position, and SmDPP11 and PgDPP11 prefer the acidic amino acids at the P1 position. This indicates that the S2 subsite can be an influential target to develop the universal inhibitor of S46 peptidases.

These substrate specificities may be helpful in bacterial growth. For $S$. maltophilia, methionine is required for growth by almost all strains ${ }^{3,28}$. SmDPP7 exhibited Met(P2) preference higher than that of PgDPP7 (Table 1), while SmDPP11 did not show Met(P2) preference (Fig. 2c). Because S. maltophilia K279a has methionine aminopeptidase ${ }^{29}$, the Met(P2) specificity of SmDPP7 may play a critical role in getting Met from peptide for the growth of this bacterium. For $P$. gingivalis, dicarboxylic amino acids (i.e., Asp and $\mathrm{Glu}$ ) in particular are needed to produce acetate, butylate, and propionate, which are short-chain fatty acids (SCFA) important for the growth and pathogenesis ${ }^{14,30-32}$. Hence, our results support the finding that PgDPP11 associated with Asn(P2) and Asp/Glu(P1) specificity ${ }^{20}$ is useful for the growth and pathogenesis of this bacterium.

The structures of four dipeptide complexes and results of ITC experiments clarify the dipeptide binding of SmDPP7 (Figs. 5 and 6). The binding modes of all dipeptide (reaction products) to SmDPP7 were enthalpy driven and were stabilised through the hydrogen bonds for $\mathrm{N}$-terminus recognition by the side chains of Asn213, Trp214, Asp672 and Asn328 (Fig. 3c) and P1-NH group recognition by the carbonyl oxygen of Phe671 (Fig. 3d). In addition, an open-to-closed conformational change would occur for SmDPP7 when it recognises dipeptides as observed in PmDAP BII (Fig. 4c), which has 78.4\% amino acid sequence identity with that of SmDPP7. Generally, recognition of a ligand molecule by strong hydrogen

Page $13 / 30$ 
bonds concomitant with large-scale conformational change is mainly enthalpy driven and unfavourable in entropy ${ }^{33}$. In addition, the loss of conformational degrees of freedom is also entropically unfavourable for the bound ligand molecule. This is the case for SmDPP7 - it recognises dipeptides by a tight hydrogen bond network (Fig. $3 \mathrm{c}$ and d) associated with open-to-closed conformational change. The order of dissociation constants $\left(K_{d}\right)$ of dipeptide ( $P 2$ a.a.; $V>N>Y>L>F$ ) was consistent with the result of competitive inhibition assay (Fig. 1 and Table S3). Among the dipeptide complexes, observations, such as strong enthalpic interactions of SmDPP7 with Asn-Tyr can be explained by an extensive hydrogenbonding network with water molecules (Figs. $5 \mathrm{~d}$ and 6). On the other hand, trapped water molecules in the $\mathrm{S} 2$ subsite are not favourable in entropy, so the Asn-Tyr binding exhibits highly unfavourable entropic contribution in ITC data (Fig. 6 and Table S3). Bindings with Tyr-Tyr, Leu-Tyr, and Phe-Tyr showed favourable enthalpic and unfavourable entropic contributions. The magnitudes of both parameters are smaller than those of Asn-Tyr binding. These thermodynamic parameters are attributed to hydrogen bonds for the dipeptide binding associated with open-to-closed conformational change. For the binding of Tyr-Tyr, Leu-Tyr, and Phe-Tyr, the ejecting water molecules from the S2 subsite as an entropy reservoir is entropically favourable. Thus totally unfavourable entropic contributions of the bindings of Tyr-Tyr, Leu-Tyr, and Phe-Tyr are explained by the fact that the favourable entropic contribution by the elimination of water molecules in the $\mathrm{S} 2$ subsite is smaller than the unfavourable entropic contributions by the opento-closed conformational change and the loss of flexibility of the bound ligand molecule, which is at the same level for the four dipeptide used in this study. Indeed, ejecting water molecules were observed for Tyr-Tyr and Phe-Tyr complexes as compared with Asn-Tyr complex (Fig. 5). Binding with Leu-Tyr, which has a hydrophobic side chain at the P2 position, could also eject water molecules from the S2 subsite. In order to clarify the detail of entropic contribution, it is necessary to measure the heat capacity changes $\left(\Delta \mathrm{C}_{\mathrm{p}}\right)$ and deconvolute total binding entropy $\left(\Delta \mathrm{S}_{\text {tot }}\right)$. Although the structure of the Tyr-Tyr complex showed hydrogen bonds to the carbonyl oxygens of Phe207 and Gly209 and the side chain of Arg218 via a water molecule (Fig. 5a), the enthalpic $\square$ contribution showed little difference as compared with Phe-Tyr binding. This suggested that this hydrogen bond does not significantly contribute to $\operatorname{Tyr}(\mathrm{P} 2)$ binding. To summarise, the dipeptide binding mode focused on the S2 subsite of SmDPP7, the P2-Asn is recognised by the establishment of hydrogen-bonding network at the bottom of the S2 subsite, and the bulky hydrophobic amino acids are accommodated in the $\mathrm{S} 2$ subsite by the hydrophobic interaction associated with the ejection of water molecules.

For DPP11 from Porphyromonas endodontalis (PeDPP11), which is a DPP11 type of S46 peptidase, the Leu-Asp (product) binding mode of PeDPP11 is energetically favourable both in enthalpy and entropy, in which enthalpic contribution is dominant ${ }^{19}$. The binding mode includes a hydrogen-bond network involving the $\mathrm{N}$-terminus and acidic residue(P1) recognition, and the ejection of solvent molecules from the inter-domain cleft concomitant with domain motion ${ }^{19}$. For the entropic contribution in the Leu-Asp binding of PeDPP11, the unfavourable entropic contribution concomitant with the structural change is completely offset by the favourable entropic contribution attributed to the ejecting water molecules, and the total entropic contribution is favourable. Although the substrate main chain recognition mechanism of PeDPP11 is similar to that of SmDPP7, the thermodynamic parameters observed for dipeptide binding 
in ITC measurements are distinct from that of SmDPP7. For SmDPP7, the unfavourable entropic $\square$ contribution in dipeptide binding indicated that an unfavourable entropic $\square$ contribution by conformational changes is more dominant than the favourable entropic $\square$ contribution by ejecting water molecules from inter-domain cleft as an entropy reservoir. It is possible to classify DPP7 and DPP11 according to the entropic $\square$ contribution in substrate binding other than the substrate specificity at the P1 position. The DPP7 type of S46 peptidases show an unfavourable entropic contribution that is attributed mainly to an open-to-closed conformational change, and the DPP11 type of S46 peptidases show a favourable entropic $\square$ contribution that is attributed to ejecting solvent molecules from the inter-domain cleft.

As described above, a universal inhibitor of S46 peptidases is desirable to optimise for the S2 subsite. In this study, we observed that the binding constant increased in proportion to the hydrophobicity and the size of the residue at the P2 position (Figs. 1 and 6). This indicates that the replacement of water molecules at the S2 subsite contributes to increasing the $\square$ binding affinity to ligands associated with favourable enthalpic and entropic $\square$ contributions. Enthalpically optimised inhibitors avoid bacteria with emerging resistance caused by mutation of targeting protein ${ }^{34,35}$. For NFGNB, mutational resistance to most antibiotics classes can arise easily ${ }^{36}$. Given these reports, compounds with favourable enthalpic contribution are convenient for the development of NFGNB antibiotics targeting S46 peptidases. In addition, the bacterial proton-dependent oligopeptide transporter (POT), a transport protein existing on the inner membrane, reportedly has substrate specificity against hydrophobic di- or tri-peptides ${ }^{37,38}$. We infer that hydrophilic compounds are effective in inhibiting $\$ 46$ peptidases that generally exist in the periplasmic space. Therefore, a compound that replaces water molecules in the S2 subsite with low LogP values may be a valuable universal inhibitor of S46 peptidase for antibiotics of NFGNB such as $S$. maltophilia and $P$. gingivalis.

In this study, we discovered the exceptional bonding of the P2 amino acid of SmDPP7, S46 peptidases, with Asn(P2), and unravelled the substrate recognition mechanisms of the $S 46$ peptidases. Asn(P2) is recognised by a hydrogen-bonding network, and hydrophobic residues are accepted by hydrophobic interactions associated with ejecting water molecules from the S2 subsite. Our findings contribute toward the development of a dipeptidyl universal inhibitor of $\$ 46$ peptidases, which could potentially serve as NFGNB antibiotics.

\section{Materials And Methods}

\section{Overexpression and purification of SmDPP7 WT and mutants.}

A synthetic gene coding for full-length SmDPP7 (residues 1-720), codon-optimized for expression in $E$. coli, was purchased from Genscript (Piscataway, USA). The target sequence corresponding to mature SmDPP7 containing the signal peptide of DAP BIII ${ }^{39}$ from Pseudoxanthomonas mexicana WO24 was cloned into the pET22b expression plasmid (Merck, Darmstadt, Germany). The mature SmDPP7 was composed of 698 amino acids (residues 23 to 720), with a theoretical molecular weight of 75720.95 and 
an isoelectric point of 8.08. Plasmids for expression of mutants, K206A, R218A, R218Q, R218K, T220A, and F671A, were obtained with overlap extension PCR using wild type expression plasmid as a template (Table S5). E. coli BL21 Gold (DE3) cells (Agilent Technologies, Santa Clara, USA) transformed with the pET22b-SmDPP7 WT and mutants expression plasmid were grown in TB media at $298 \mathrm{~K}$ to an $\mathrm{OD}_{600}$ of 0.6. Overproductions of SmDPP7 WT and mutants were carried out by adding $0.1 \mathrm{mM}$ Isopropyl- $\beta$-Dthiogalactopyranoside for $15 \mathrm{~h}$ at $298 \mathrm{~K}$. Thereafter, the cells were harvested by centrifugation at $6,000 \mathrm{x}$ g. Cells were disrupted using sonication and the cell extract was obtained by centrifuging the lysate at $20,000 \times \mathrm{g}$ for $30 \mathrm{~min}$. The SmDPP7 WT and mutants were purified by precipitation with 35 to $70 \%$ ammonium sulfate and hydrophobic column chromatography using a HiPrep 16/10 Butyl column (Cytiva, Marlborough, MA, USA). The eluate was desalted using a HiPrep 26/10 desalting column (Cytiva) and finally subjected to anion-exchange column chromatography using a Mono Q 5/50 GL column (Cytiva). The fractions containing SmDPP7 WT and mutants were pooled, buffer-exchanged to $80 \mathrm{mM}$ Tris/HCl pH 8.5 and concentrated to $5 \mathrm{mg} / \mathrm{ml}$ using Vivaspin 20 concentrator (Cytiva). Protein concentration was determined by Bradford assay, and a linear calibration curve was obtained using bovine gamma-globulin ranging from 0 to $0.25 \mathrm{mg} / \mathrm{ml}$.

\section{Overexpression and purification of PgDPP7, SmDPP11 and PgDPP11}

Synthetic genes coding for full-length PgDPP7 (residues 1-712, UniProt accession number Q7MWU6) and SmDPP11 (residues 1-715, UniProt accession number B4SNQ8), codon-optimized for expression in E. coli, were purchased from Genscript (NJ, USA). The target sequences corresponding to mature PgDPP7 (Asp25-Ile712) and mature SmDPP11 (Asp24-GIn715), both containing the signal peptide of DAP BIII ${ }^{39}$ from $P$. mexicana WO24 were amplified using PCR and cloned into the PET22b expression plasmid (Merck, Darmstadt, Germany). Overproduction and purification of PgDPP7 and SmDPP11 were performed in a way similar to those for SmDPP7 described above. Overproduction and purification of PgDPP11 has been described in the literature ${ }^{17}$.

\section{Determination of kinetic parameters toward dipeptidyl MCA}

Kinetic parameters were determined by fitting the experimental data to the Michaelis-Menten equation using Excel Solver (Microsoft, USA) by nonlinear least-squares fitting with different concentrations of glycyl-I-tyrosine-4-methylcoumaryl-7-amide $(1.56,3.13,6.25,12.5,25,50$, and $100 \mu \mathrm{M}$; Gly-Tyr-MCA; scrum) and I-tyrosine-l- tyrosine-4-methylcoumaryl-7-amide $(3.13,6.25,12.5,25,50,100$, and $200 \mu \mathrm{M}$; TyrTyr-MCA; scrum) as a substrate. The enzyme reaction was performed in a reaction buffer consisting of 50 $\mathrm{mM}$ sodium phosphate buffer $\mathrm{pH}$ 7.0, $5 \mathrm{mM}$ EDTA and $0.005 \%$ Tween 20 at $298 \mathrm{~K}$ for $20 \mathrm{~min}$ and standard deviations were calculated from three independent experiments. The fluorescence intensity of the released MCA was measured with excitation at $355 \mathrm{~nm}$ and emission at $460 \mathrm{~nm}$ using an Infinite 200 PRO microplate reader (Tecan, Switzerland).

\section{Competitive inhibition assay using dipeptide library}


The dipeptide library was purchased from Funakoshi Co., Ltd. (Tokyo, Japan). Different concentrations $(3.13,6.25,12.5,25,50,100$, and $200 \mu \mathrm{M})$ of dipeptides as the inhibitor and $200 \mu \mathrm{M}$ Tyr-Tyr-MCA (PEPTIDE INSTITUTE, Osaka, Japan) as the substrate for DPP7 were added into a reaction buffer consisting of $50 \mathrm{mM}$ sodium phosphate buffer $\mathrm{pH}$ 7.0, $5 \mathrm{mM}$ EDTA and 0.005\% Tween 20. For DPP11s, different concentrations $(1.56,3.13,6.25,12.5,25,50$, and $100 \mu \mathrm{M})$ of dipeptides as the inhibitor and 100 $\mu \mathrm{M}$ Leu-Asp-MCA (Peptide Institute, Japan) as the substrate were used. The enzyme reaction was performed at $298 \mathrm{~K}$ for $20 \mathrm{~min}$ and standard deviations were calculated from three independent experiments. Half-maximal inhibitory concentration $\left(\mathrm{IC}_{50}\right)$ values were determined by fitting to a sigmoid curve (4-parameter logistic curve) using ImageJ software ${ }^{40}$ and inhibition constants $\left(K_{\mathrm{i}}\right)$ were calculated using the Cheng-Prusoff equation ${ }^{41}$. The fluorescence intensity of the released MCA was measured with the same method of determination of kinetics parameters toward dipeptidyl MCA.

\section{Crystallisation of SmDPP7}

Crystals of the SmDPP7/dipeptide complexes were prepared as follows: Asn-Tyr and Val-Tyr-Pro were purchased from GenScript (Piscataway, NJ, USA). Phe-Tyr and Tyr-Tyr-Tyr were purchased from SigmaAldrich (St Louis, MO, USA) and Santa Cruz Biotech (Dallas, TX, SUA) respectively. The tripeptides, ValTyr-Pro and Tyr-Tyr-Tyr (tripeptides were used as a substrate, and the N-terminal dipeptide was observed as the bound product as described below), were dissolved in $80 \mathrm{mM}$ Tris- $\mathrm{HCl}, \mathrm{pH} 8.5$ to concentrations of $30.0 \mathrm{mM}$ and $20.0 \mathrm{mM}$, respectively. The $5-\mathrm{mg} / \mathrm{ml} \mathrm{SmDPP7}$ solution was mixed with aliquots of the respective ligand solutions at a volume ratio of 9:1 for the tripeptides Val-Tyr-Pro and Tyr-Tyr-Tyr, with final ligand concentrations of $3.0 \mathrm{mM}$ and $2.0 \mathrm{mM}$, respectively. The samples were crystallised using the hanging-drop method; $1 \mu \mathrm{l}$ of protein solution was mixed with the same volume of reservoir solution (20\% (w/v) PEG8000 and $0.2 \mathrm{M}$ ammonium acetate) and incubated at $293 \mathrm{~K}$. The drops were suspended over $200 \mu \mathrm{l}$ of reservoir solution in 48-well plates.

Asn-Tyr and Phe-Tyr were dissolved in purified water to concentrations of $100 \mathrm{mM}$. The $5-\mathrm{mg} / \mathrm{ml}$ SmDPP7 solution was mixed with aliquots of the respective ligand solutions at a volume ratio of 25:1 for the dipeptides Asn-Tyr and Phe-Tyr, with a final ligand concentration of $4.0 \mathrm{mM}$. The samples were crystallised using the hanging-drop method; $0.95 \mu \mathrm{l}$ of protein solution was mixed with the same volume of reservoir solution (20\%(w/v) PEG8000 and $0.2 \mathrm{M}$ Calcium acetate) and incubated at $293 \mathrm{~K}$. The drops were suspended over $200 \mu \mathrm{l}$ of reservoir solution in 48-well plates.

A dipeptide complex, Tyr-Tyr complex, was obtained by co-crystallisation of SmDPP7 with a tripeptide Tyr-Tyr-Tyr, because the tripeptide Tyr-Tyr-Tyr was commercially available at a lower cost as compared with custom peptide synthesis of the dipeptide Tyr-Tyr. For the Tyr-Tyr complex, clear continuous electron density was observed for the first two residues of the tripeptide (Figure S2a), and no clear electron density was observed for the last residue. Because the SmDPP7 enzyme reaction occurred in the solution used for crystallisation, the Tyr-Tyr-Tyr (P2-P1-P1') tripeptide acted as the substrate, and the reaction products were the N-terminal Tyr-Tyr (P2-P1) dipeptide and the $\mathrm{C}$-terminal Tyr (P1'). While the $\mathrm{N}$-terminal product Tyr-Tyr remained at the active site, the $\mathrm{C}$-terminal product Tyr dissociated from the active site. The 
asymmetric unit of the Tyr-Tyr complex was composed of two independent SmDPP7 subunits; in both subunits (Figure 3a), the hydrolysed dipeptide product $\left(\mathrm{NH}_{2}-\mathrm{Tyr}-\mathrm{Tyr}-\mathrm{COOH}\right)$, rather than a reaction intermediate, was observed (Figure S2a). Similarly, another dipeptide complex, Val-Tyr complex, was obtained by co-crystallisation of SmDPP7 with a tripeptide Val-Tyr-Pro and the hydrolysed dipeptide product $\left(\mathrm{NH}_{2}\right.$-Val-Tyr- $\mathrm{COOH}$ ) was observed (Figure $\left.\mathbf{S} 2 \mathbf{b}\right)$. In this case, co-crystallisation with Val-Tyr-Pro was performed to confirm the ability of imino-bond (X-Pro) hydrolysis by SmDPP7, though the dipeptide Val-Tyr was commercially available at a reasonable cost. The other dipeptide complexes Phe-Tyr and Asn-Tyr complexes, were obtained by co-crystallisation of SmDPP7 with dipeptides Phe-Tyr and Asn Tyr, respectively (Figures S2c and S2d).

\section{X-ray data collection}

For data collection under cryogenic conditions, dipeptide-complex crystals in a droplet were directly transferred to harvesting solutions [16\%(w/v) PEG8000, $0.16 \mathrm{M}$ ammonium acetate and $20 \%(\mathrm{v} / \mathrm{v})$ glycerol] and [16\%(w/v) PEG8000, 0.16 M calcium acetate and 20\%(v/v) glycerol] respectively for 10 seconds. Crystals were mounted in nylon loops or MicroMounts ${ }^{\mathrm{TM}}$ (MiTeGen, Ithaca, NY, USA) and flashcooled in a cold nitrogen gas stream at $100 \mathrm{~K}$ immediately before data collection. Data were collected by the rotation method at $100 \mathrm{~K}$ using a MAR300HE CCD detector or EIGER $16 \mathrm{M}$ detector with synchrotron radiation source on the beamline BL44XU at SPring-8. Laue group and unit-cell parameters were determined using the xia2/DIALS software package ${ }^{42}$ with XDS $^{43}$ or MOSFLM ${ }^{44}$. The cell parameters and data-collection statistics are summarised in Table S1.

\section{Structure determination}

The initial phase determination was performed for the Val-Tyr complex of SmDPP7 using the molecular replacement method. One protomer of PmDAP BII ${ }^{18}$ (PDB code: 3WOL), which has approximately $78 \%$ amino-acid sequence identity to SmDPP7, was used as a search model. Cross-rotation and translation functions were calculated using the MOLREP program ${ }^{45}$ from CCP4 suite ${ }^{46}$. Structure refinement was carried out with the program REFMAC $5^{47}$, and further iterative manual model building and refinement were performed using the programs $\mathrm{Coot}^{48}$ and REFMAC5 ${ }^{47}$. The stereochemistry of the model was verified using RAMPAGE ${ }^{49}$ and PROCHECK ${ }^{50}$ programs. The refined structure of the Val-Tyr complex was then used for the structural determination of the Tyr-Tyr complex by the difference Fourier method The refined structure of the Tyr-Tyr complex was used for the initial phase determination of Asn-Tyr and PheTyr complexes. Cross-rotation and translation functions were calculated using the program PHASER ${ }^{51}$ from the CCP4 suite ${ }^{46}$. After the final round of refinement, the bound dipeptide molecules were removed from the model. Then, the amplitude $|F \mathrm{c}|$ and phase angles calculated from the partial structure were

used to calculate a weighted $m\left|F_{0}\right|-D\left|F_{\mathrm{C}}\right|$ omit map ${ }^{47}$, where ' $m$ ' is the figure of merit (approximately equal to the cosine of the phase error) and ' $D$ ' is the estimate of the coordinate error in the partial structure (Figure S2). The refinement statistics are summarised in Table S2.

\section{Isothermal Titration Calorimetry}

Page $18 / 30$ 
The bindings were analysed using a MICROCAL PEAQ-ITC microcalorimeter (Malvern, UK). The binding reactions were performed in $50 \mathrm{mM}$ sodium phosphate $\mathrm{pH} 7.0$ and $2.5 \%$ dimethyl sulfoxide at $25^{\circ} \mathrm{C}$ and were stirred at $750 \mathrm{rpm}$. A single injection of $0.4 \mu \mathrm{l}$ and 19 times injections of $2.0 \mu \mathrm{l}$ of the dipeptide solution were injected into $350 \mu$ of enzyme solution (wild-type SmDPP7). The wild-type SmDPP7 concentration was $25 \mu \mathrm{M}$ and the concentration of each dipeptide solution was $250 \mu \mathrm{M}$ in reaction buffer. Each injection was performed for 4 seconds with an interval of $150 \mathrm{sec}$ between injections. The dissociation constant $\left(K_{\mathrm{d}}\right)$ and heats of binding $(\Delta H)$ were obtained using MICROCAL PEAQ-ITC Analysis software (Malvern, UK). Gibbs free energy $(\Delta G)$ values were calculated according to $\Delta G=-R T \ln K_{\mathrm{a}}=R T$ In $K_{\mathrm{d}}\left(K_{\mathrm{a}}=1 / K_{\mathrm{d}}\right.$, association constants).

\section{Graphical programs.}

Figures 3 to 6, S2 and S4 were produced using the program UCSF Chimera ${ }^{52}$. Figure S3 was created using Adobe Illustrator (Adobe Systems Inc., San Jose, USA).

\section{Declarations}

\section{Competing financial interests}

The authors declare that they have no competing financial interests to disclose.

\section{Acknowledgements}

We thank Drs. Y. Yamada and N. Matsugaki of the Photon Factory and Drs. A. Higashiura, K. Hasegawa, K. Takagi, K. Sakurai and E. Yamashita of SPring-8 for their help with data collection at the synchrotron facilities, and Drs. H. Narita, M. Matsuda and A. Nakagawa of Osaka university for their help with ITC experiment. We also thank Dr. K. Tsutsumi and A. Matsumoto of Osaka university for their help with Protein Crystallisation. The synchrotron radiation experiments were performed at BL-1A, BL-17A and NE3A of the Photon Factory (Proposal Nos. 2011G090, 2013G138 and 2017G162) and at BL41XU and BL44XU of SPring-8 (Proposal Nos. 2013A6822, 2013B6822, 2014A6924, 2014B6924, 2015A6521, 2015B6521, 2016B6620, 2017A6721, 2017B6721, 2018A6818, 2018B6818, 2019A6917 and 2019B6917). This research was supported by a Grants-in-Aid for Scientific Research(C) 16K08322 (to Yasu S.) and Grants-in-Aid for Scientific Research(B) $17 \mathrm{H} 03790$ (to W.O.) and 16H04902 and $19 \mathrm{H} 02876$ (to N.T.) from the MEXT, the Platform Project for Supporting in Drug Discovery and Life Science Research from the AMED (to Yasu S. and N.T.), a grant from the Takeda Science Foundation (to Yasu S.), the Collaborative Researcher Program of the Institute for Protein Research, Osaka University (Proposal Nos. CR1405, CR1505, CR1605, CR1705, CR1805 and CR1905) (to N.T. and Yasu S.), Private University Research Branding Project $\triangle \mathrm{H} 29-\mathrm{A} 3 \mathrm{Z}$ (to Yasu S.), and a Nagai Memorial Research Scholarship from the Pharmaceutical Society of Japan (to R.S.). This study was also supported in part by "High-Quality Protein Crystal Growth Experiment on JEM" promoted by JAXA (Japan Aerospace Exploration Agency) (to Yasu 
S.). The Russian Spacecraft "Progress" and "Soyuz" provided by the Russian Federal Space Agency were used for space transportation. Some of the space crystallisation technology was developed by ESA (the European Space Agency) and the University of Granada.

\section{Author contributions}

A.N. cloned the construct, performed biochemical analyses, analysed the data, and wrote the paper; Yoshi S. performed biochemical analyses, analysed the data, and wrote the paper; Yasu S. designed the study, crystallised the protein, collected X-ray data, analysed the data and wrote the paper; S.R., C.K., N.Y., and M.T. crystallised the protein, collected X-ray data, analysed the data; Yosuke S. performed the biochemical analyses; H.G. and T.N. designed the study and analysed the data; N.T. and W.O. designed the study, analysed the data, and wrote the paper. All authors discussed the results and commented on the manuscript.

\section{References}

1. Farha, M. A. \& Brown, E. D. Drug repurposing for antimicrobial discovery. Nat. Microbiol. 4, 565-577 (2019).

2. El Chakhtoura, N. G. et al. Therapies for multidrug resistant and extensively drug-resistant nonfermenting gram-negative bacteria causing nosocomial infections: a perilous journey toward 'molecularly targeted' therapy. Expert Rev. Anti. Infect. Ther. 16, 89-110 (2018).

3. Palleroni, N. J. \& Bradbury, J. F. Stenotrophomonas, a New Bacterial Genus for Xanthomonas maltophilia (Hugh 1980) Swings et al. 1983. Int. J. Syst. Bacteriol. 43, 606-609 (1993).

4. Brooke, J. S. Stenotrophomonas maltophilia: An emerging global opportunistic pathogen. Clin. Microbiol. Rev. 25, 2-41 (2012).

5. Chang, Y. T., Lin, C. Y., Chen, Y. H. \& Hsueh, P.-R. Update on infections caused by Stenotrophomonas maltophilia with particular attention to resistance mechanisms and therapeutic options. Frontiers in Microbiology 6, 893 (2015).

6. Falagas, M. E. et al. Attributable mortality of Stenotrophomonas maltophilia infections: a systematic review of the literature. Future Microbiol. 4, 1103-1109 (2009).

7. Spencer, R. C. The emergence of epidemic, multiple-antibiotic-resistant Stenotrophomonas (Xanthomonas) maltophilia and Burkholderia (Pseudomonas) cepacia. J. Hosp. Infect. 30, 453-464 (1995).

8. Fujita, J. et al. Clinical features of Stenotrophomonas maltophilia pneumonia in immunocompromised patients. Respir. Med. 90, 35-38 (1996).

9. Brooke, J. S. New strategies against Stenotrophomonas maltophilia: a serious worldwide intrinsically drug-resistant opportunistic pathogen. Expert Rev. Anti. Infect. Ther. 12, 1-4 (2014). 
10. Al-Anazi, K. A. \& Al-Jasser, A. M. Infections Caused by Stenotrophomonas maltophilia in Recipients of Hematopoietic Stem Cell Transplantation. Frontiers in Oncology 4, 232 (2014).

11. Suzuki, Y. et al. Identification of the Catalytic Triad of Family S46 Exopeptidases, Closely Related to Clan PA Endopeptidases. Sci. Rep. 4, 1-10 (2014).

12. Nemoto, T. K. et al. Identification of a new subtype of dipeptidyl peptidase 11 and a third group of the S46-family members specifically present in the genus Bacteroides. Biochimie 147, 25-35 (2018).

13. Ohara-Nemoto, Y. et al. Identification and characterization of prokaryotic dipeptidyl-peptidase 5 from Porphyromonas gingivalis. J. Biol. Chem. 289, 5436-5448 (2014).

14. Takahashi, N., Sato, T. \& Yamada, T. Metabolic pathways for cytotoxic end product formation from glutamate- and aspartate-containing peptides by Porphyromonas gingivalis. J. Bacteriol. 182, 47044710 (2000).

15. Sakamoto, Y. et al. Fragment-based discovery of the first nonpeptidyl inhibitor of an S46 family peptidase. Sci. Rep. 9, 13587 (2019).

16. Rouf, S. M. A. et al. Discrimination based on Gly and Arg/Ser at position 673 between dipeptidylpeptidase (DPP) 7 and DPP11, widely distributed DPPs in pathogenic and environmental gramnegative bacteria. Biochimie 95, 824-832 (2013).

17. Sakamoto, Y. et al. Structural and mutational analyses of dipeptidyl peptidase 11 from Porphyromonas gingivalis reveal the molecular basis for strict substrate specificity. Sci. Rep. 5, 11151 (2015).

18. Sakamoto, Y. et al. S46 peptidases are the first exopeptidases to be members of clan PA. Sci. Rep. 4, 4977 (2014).

19. Bezerra, G. A. et al. Bacterial protease uses distinct thermodynamic signatures for substrate recognition. Sci. Rep. 7, 2848 (2017).

20. Ohara-nemoto, Y. et al. Asp- and Glu-specific novel dipeptidyl peptidase 11 of Porphyromonas gingivalis ensures utilization of Proteinaceous energy sources. J. Biol. Chem. 286, 38115-38127 (2011).

21. Rouf, S. M. A. et al. Phenylalanine 664 of dipeptidyl peptidase (DPP) 7 and Phenylalanine 671 of DPP11 mediate preference for P2-position hydrophobic residues of a substrate. FEBS Open Bio 3, 177-183 (2013).

22. Soisson, S. M. et al. Structural definition and substrate specificity of the $\mathrm{S} 28$ protease family: the crystal structure of human prolylcarboxypeptidase. BMC Struct. Biol. 10, 16 (2010).

23. Bezerra, G. A. et al. Structures of Human DPP7 Reveal the Molecular Basis of Specific Inhibition and the Architectural Diversity of Proline-Specific Peptidases. PLoS One 7, e43019 (2012).

24. Nemoto, T. K., Ono, T. \& Ohara-Nemoto, Y. Establishment of potent and specific synthetic substrate for dipeptidyl-peptidase 7. Anal. Biochem. 548, 78-81 (2018).

25. Sereda, T. J., Mant, C. T., Sönnichsen, F. D. \& Hodges, R. S. Reversed-phase chromatography of synthetic amphipathic a-helical peptides as a model for ligand/receptor interactions Effect of 
changing hydrophobic environment on the relative hydrophilicity/hydrophobicity of amino acid sidechains. J. Chromatogr. A 676, 139-153 (1994).

26. Monera, O. D., Sereda, T. J., Zhou, N. E., Kay, C. M. \& Hodges, R. S. Relationship of sidechain hydrophobicity and a-helical propensity on the stability of the single-stranded amphipathic a-helix. $J$. Pept. Sci. 1, 319-329 (1995).

27. Rawlings, N. D. et al. The MEROPS database of proteolytic enzymes, their substrates and inhibitors in 2017 and a comparison with peptidases in the PANTHER database. Nucleic Acids Res. 46, D624D632 (2017).

28. Ikemoto, S., Suzuki, K., Kaneko, T. \& Komagata, K. Characterization of Strains of Pseudomonas maltophilia Which Do Not Require Methionine. Int. J. Syst. Evol. Microbiol. 30, 437-447 (1980).

29. Crossman, L. C. et al. The complete genome, comparative and functional analysis of Stenotrophomonas maltophilia reveals an organism heavily shielded by drug resistance determinants. Genome Biol. 9, R74 (2008).

30. Mazumdar, V., Snitkin, E. S., Amar, S., Segrè, D. \& Segre, D. Metabolic Network Model of a Human Oral Pathogen. J. Bacteriol. 191, 74 LP - 90 (2009).

31. Sato, M. et al. Three CoA Transferases Involved in the Production of Short Chain Fatty Acids in Porphyromonas gingivalis. Front. Microbiol. 7, 1146 (2016).

32. Takahashi, N. \& Sato, T. Dipeptide utilization by the periodontal pathogens Porphyromonas gingivalis, Prevotella intermedia, Prevotella nigrescens and Fusobacterium nucleatum. Oral Microbiol. Immunol. 17, 50-54 (2002).

33. Ferenczy, G. G. \& Keserü, G. M. Thermodynamics guided lead discovery and optimization. Drug Discov. Today 15, 919-932 (2010).

34. Freire, E. Do enthalpy and entropy distinguish first in class from best in class? Drug Discov. Today 13, 869-874 (2008).

35. Ohtaka, H., Freire, E., Ã, E. F. \& Freire, E. Adaptive inhibitors of the HIV-1 protease. Prog. Biophys. Mol. Biol. 88, 193-208 (2005).

36. Hancock, R. E. W. \& Speert, D. P. Antibiotic resistance in Pseudomonas aeruginosa: mechanisms and impact on treatment. Drug Resist. Updat. 3, 247-255 (2000).

37. Newstead, S. et al. Crystal structure of a prokaryotic homologue of the mammalian oligopeptideproton symporters, PepT1 and PepT2. EMBO J. 30, 417-426 (2011).

38. Doki, S. et al. Structural basis for dynamic mechanism of proton-coupled symport by the peptide transporter POT. Proc. Natl. Acad. Sci. 110, 11343-11348 (2013).

39. Ogasawara, W., Kobayashi, G., Okada, H. \& Morikawa, Y. Two types of novel dipeptidyl aminopeptidases from Pseudomonas sp. strain WO24. J. Bacteriol. 178, 6288-6295 (1996).

40. Schneider, C. A., Rasband, W. S. \& Eliceiri, K. W. NIH Image to ImageJ: 25 years of image analysis. Nat. Methods 9, 671 (2012). 
41. Yung-Chi, C. \& Prusoff, W. H. Relationship between the inhibition constant (KI) and the concentration of inhibitor which causes 50 per cent inhibition (150) of an enzymatic reaction. Biochem. Pharmacol. 22, 3099-3108 (1973).

42. Winter, G. et al. DIALS: implementation and evaluation of a new integration package. Acta Crystallogr. Sect. D 74, 85-97 (2018).

43. Kabsch, W. XDS. Acta Crystallogr. Sect. D 66, 125-132 (2010).

44. Leslie, A. G. W. Recent changes to the MOSFLM package for processing film and image plate data. Jt. CCP4 +ESF-EAMCB Newsl. Protein Crystallogr. 26 (1992).

45. Vagin, A. \& Teplyakov, A. Molecular replacement with MOLREP. Acta Crystallogr. Sect. D 66, 22-25 (2010).

46. The CCP4 suite: programs for protein crystallography. Acta Crystallogr. D. Biol. Crystallogr. 50, 760763 (1994).

47. Murshudov, G. N. et al. REFMAC5 for the refinement of macromolecular crystal structures. Acta Crystallogr. Sect. D 67, 355-367 (2011).

48. Emsley, P. \& Cowtan, K. Coot: model-building tools for molecular graphics. Acta Crystallogr. Sect. $D$ 60, 2126-2132 (2004).

49. Lovell, S. C. et al. Structure validation by Ca geometry: $\phi, \psi$ and $C \beta$ deviation. Proteins Struct. Funct. Bioinforma. 50, 437-450 (2003).

50. Laskowski, R. A., MacArthur, M. W., Moss, D. S. \& Thornton, J. M. PROCHECK: a program to check the stereochemical quality of protein structures. J. Appl. Crystallogr. 26, 283-291 (1993).

51. McCoy, A. J. et al. Phaser crystallographic software. J. Appl. Crystallogr. 40, 658-674 (2007).

52. Pettersen, E. F. et al. UCSF Chimera-A visualization system for exploratory research and analysis. J. Comput. Chem. 25, 1605-1612 (2004).

\section{Figures}




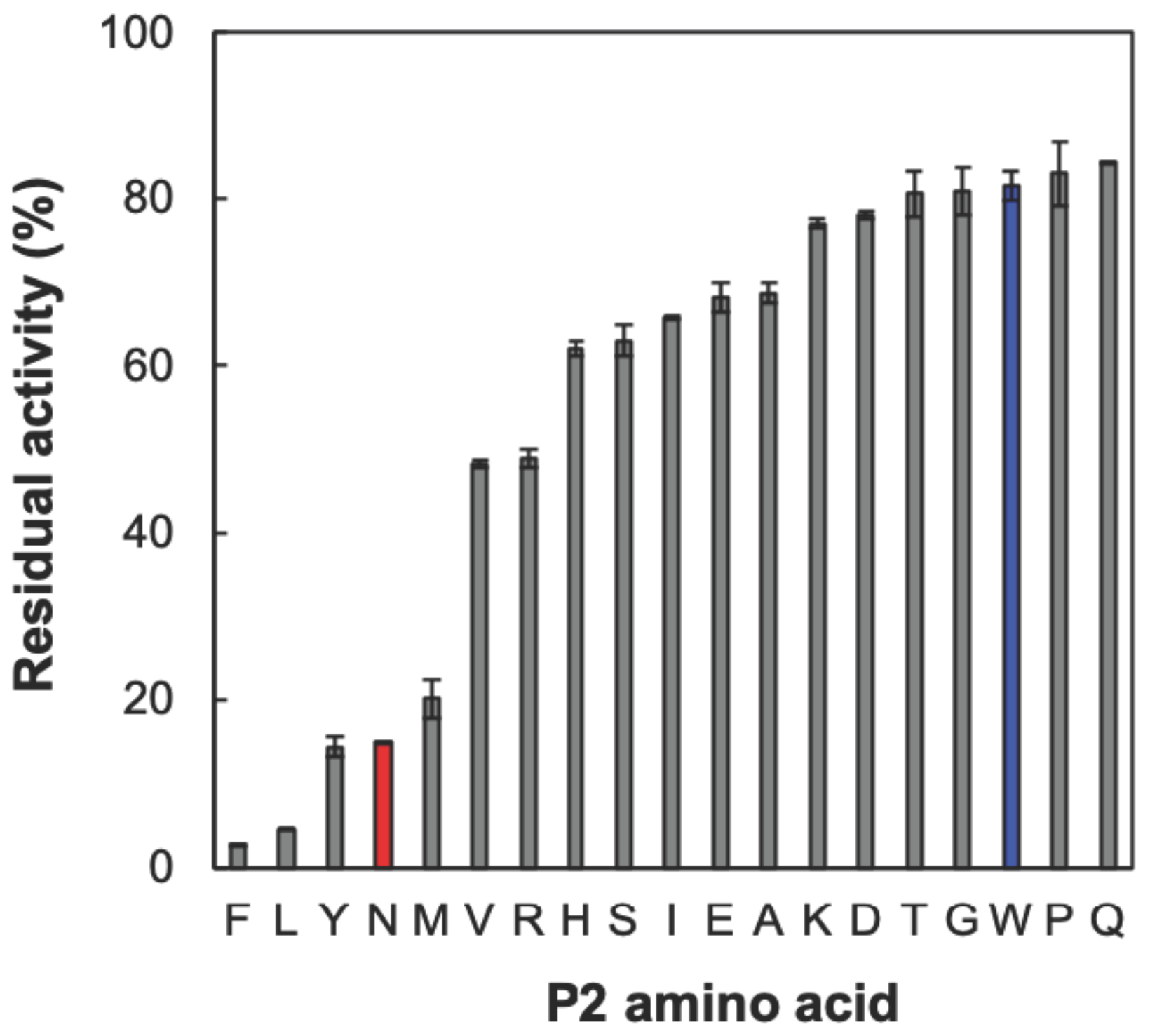

Figure 1

Competitive inhibition assay using Xaa-Tyr dipeptides against the hydrolytic activity on a synthetic substrate of SmDPP7. Descending order of the P2-preferences of SmDPP7. Tyr-Tyr-MCA hydrolysis of SmDPP7 was inhibited by Xaa-Tyr dipeptide. Residual activity was measured under conditions where 200 $\mu \mathrm{M}$ dipeptides and $200 \mu \mathrm{M}$ Tyr-Tyr-MCA were added into the reaction solution. Cys-Tyr could not be purchased commercially. The outlier dipeptides Asn-Tyr and Trp-Tyr are coloured in red and blue, respectively (see text and Figure 2a). The standard deviation was obtained from three independent experiments. 

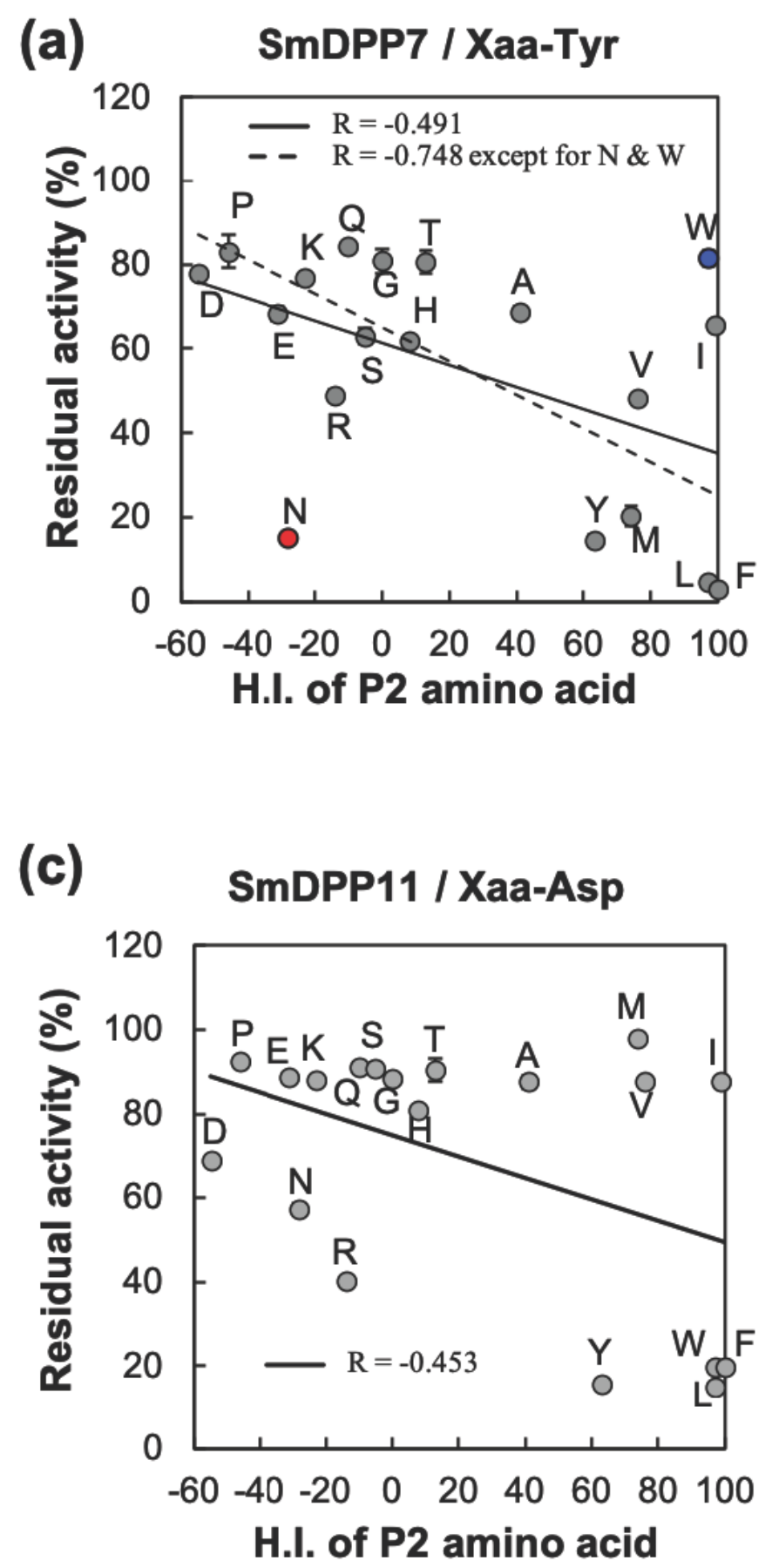

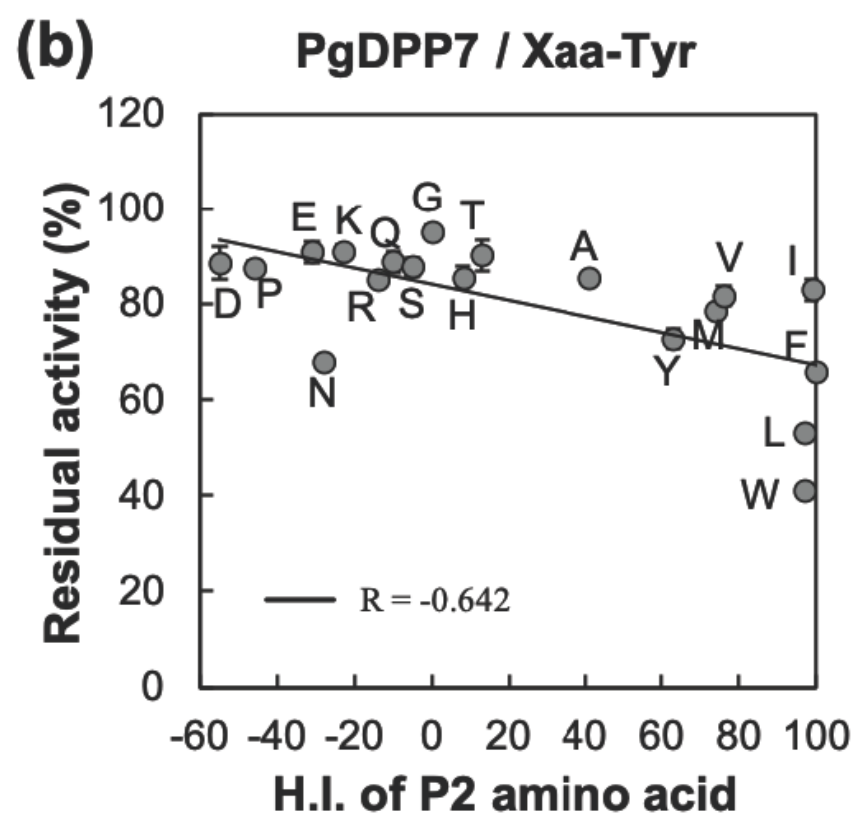

(d)

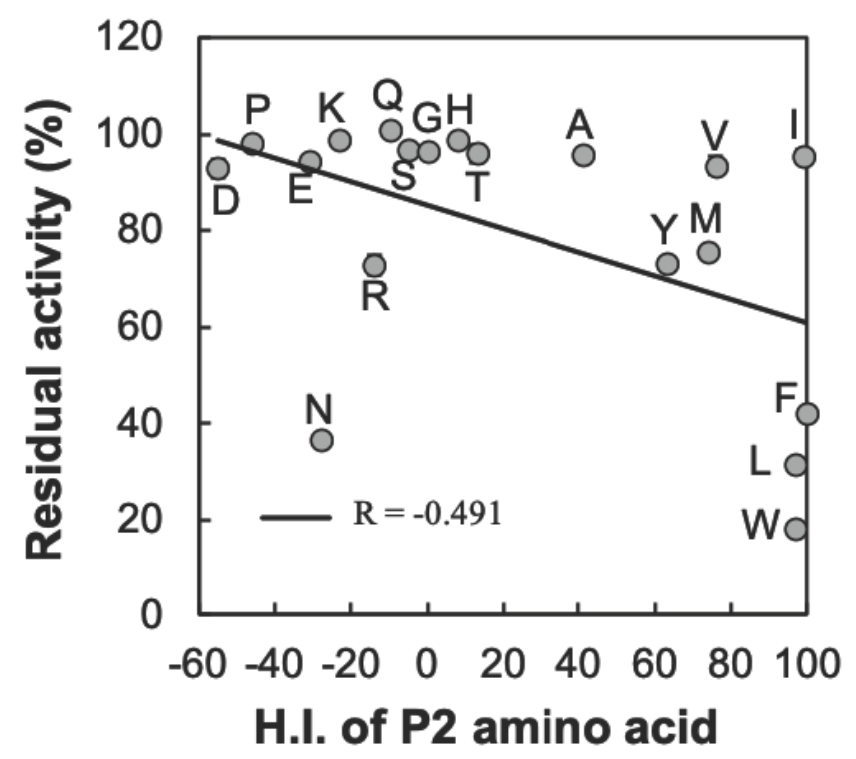

Figure 2

Correlation diagrams between the hydrophobicity index (H.I.) of P2-amino acid and residual DPP activities of S46 peptidases. (a) SmDPP7. The outlier dipeptides Asn-Tyr and Trp-Tyr are coloured in red and blue, respectively (see text). (b) PgDPP7. Tyr-Tyr-MCA hydrolysis of PgDPP7 was inhibited by Xaa-Tyr dipeptide. Residual activity was measured under conditions where $200 \mu \mathrm{M}$ dipeptides and $200 \mu \mathrm{M}$ TyrTyr-MCA were added into the reaction solution. Cys-Tyr could not be purchased commercially. (c) DPP11 from S. maltophilia (SmDPP11). (d) PgDPP11. For DPP11s, Leu-Asp-MCA hydrolysis was inhibited by Xaa-Asp dipeptide. Residual activity was measured under conditions where $100 \mu \mathrm{M}$ dipeptides and 100 
$\mu \mathrm{M}$ Leu-Asp-MCA were added into the reaction solution. Cys-Asp could not be purchased commercially. The hydrophobicity indices of the amino acids were adopted from Sereda et al., 1994 and Mohera et al. 199525,26 . $R$ is the correlation coefficient. The standard deviation was obtained from three independent experiments.
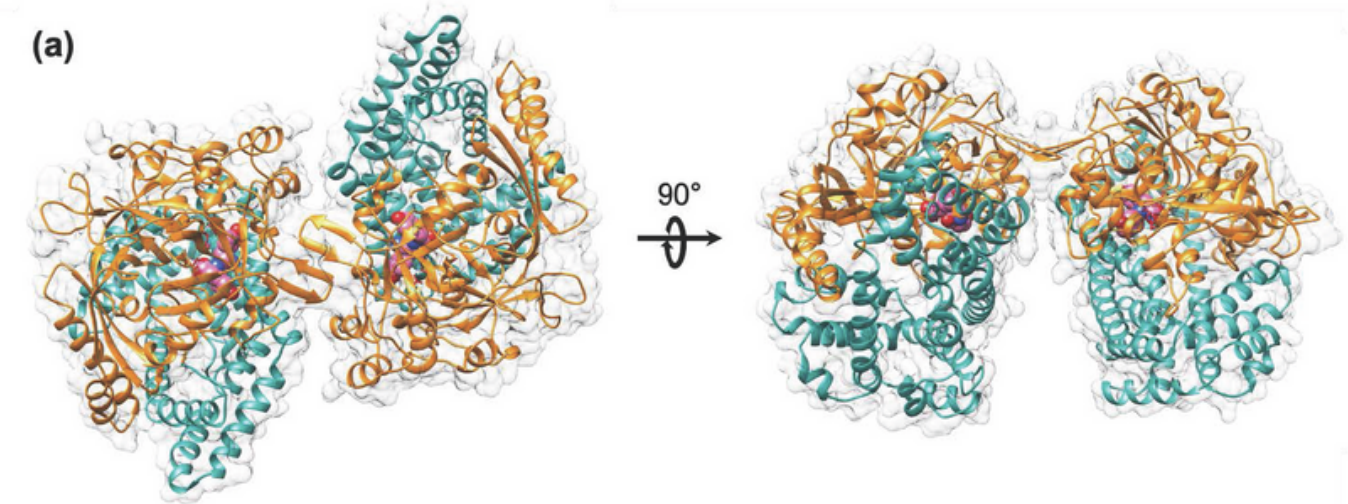

(b)
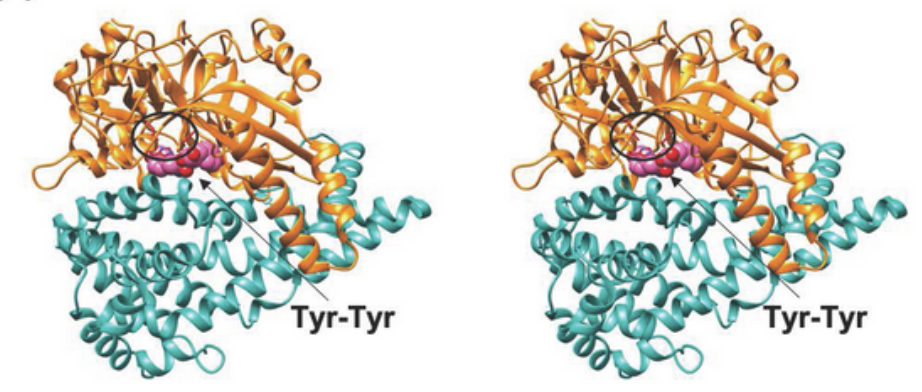

(c)

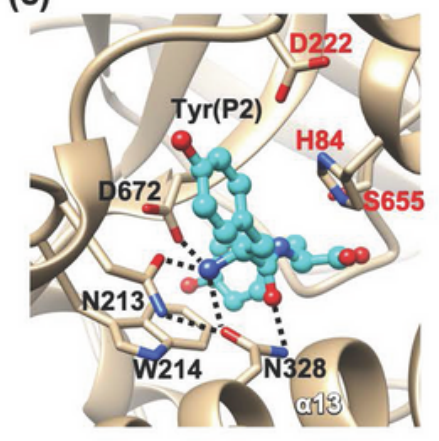

(d)

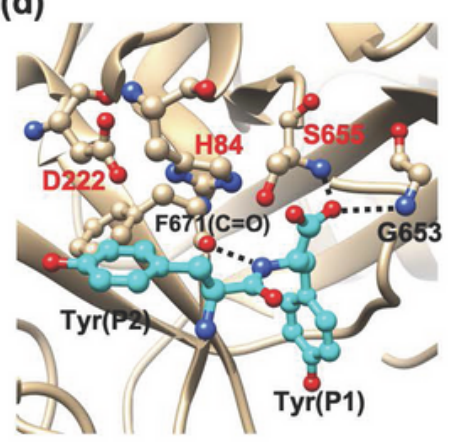

(e)

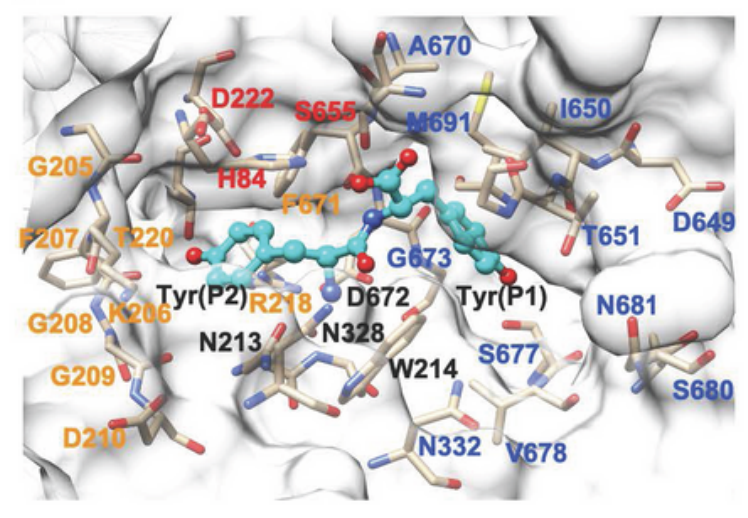

Figure 3 
Three-dimensional structure of SmDPP7 complexed with Tyr-Tyr dipeptide (a) Three-dimensional structure of dimeric SmDPP7 complexed with Tyr-Tyr dipeptide (PDB, 7DKC). Orange and sea green represent catalytic domain (residues 23-274 and 572-720) and a-helical domain (residues 275-571), respectively. The bound Tyr-Tyr dipeptide is coloured in pink. (b) Wall-eye stereo view of monomeric SmDPP7. The catalytic triad "Asp222-His84-Ser655" (red) is marked by an ellipsoid. The other colour codes are the same as in (a). (c) $\mathrm{N}$-terminus recognition. Catalytic residues and $\mathrm{N}$-terminal recognition residues are represented in red and black, respectively. (d) Oxyanion hole. Catalytic residues are represented in red. (e) S1 and S2 subsite. S1 subsite residues and S2 subsite residues are represented in blue and orange, respectively. The other colour codes are the same as in (c). 

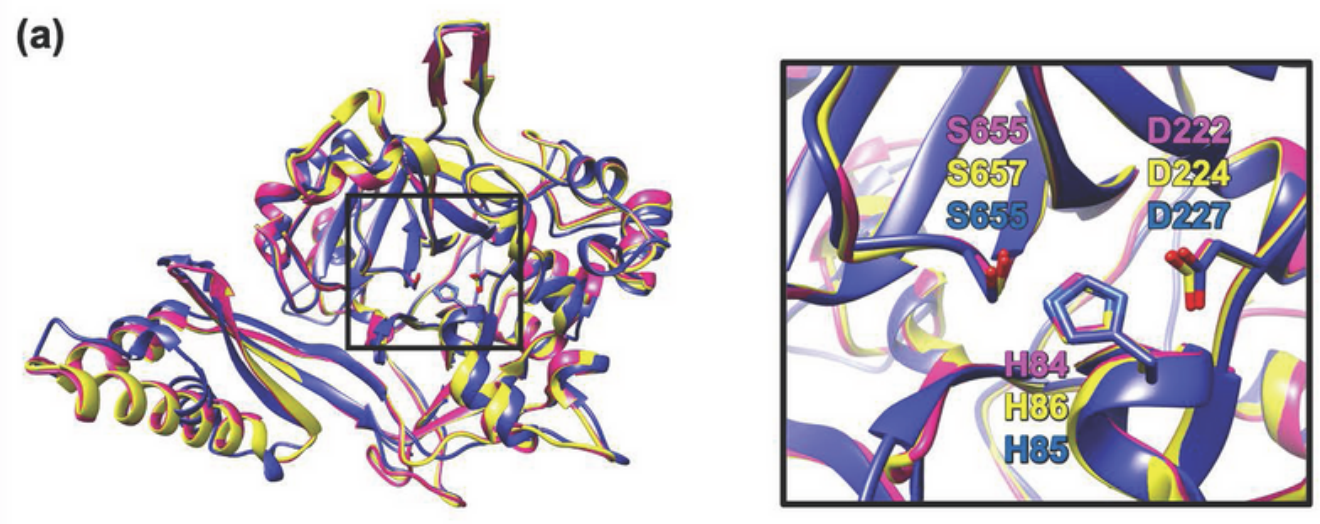

(b)

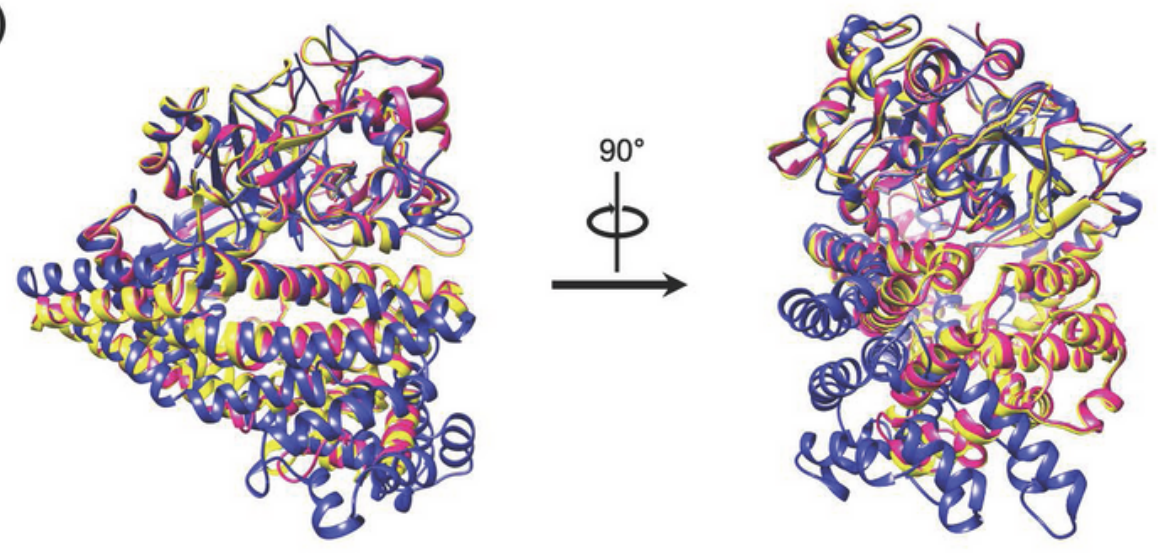

(c)
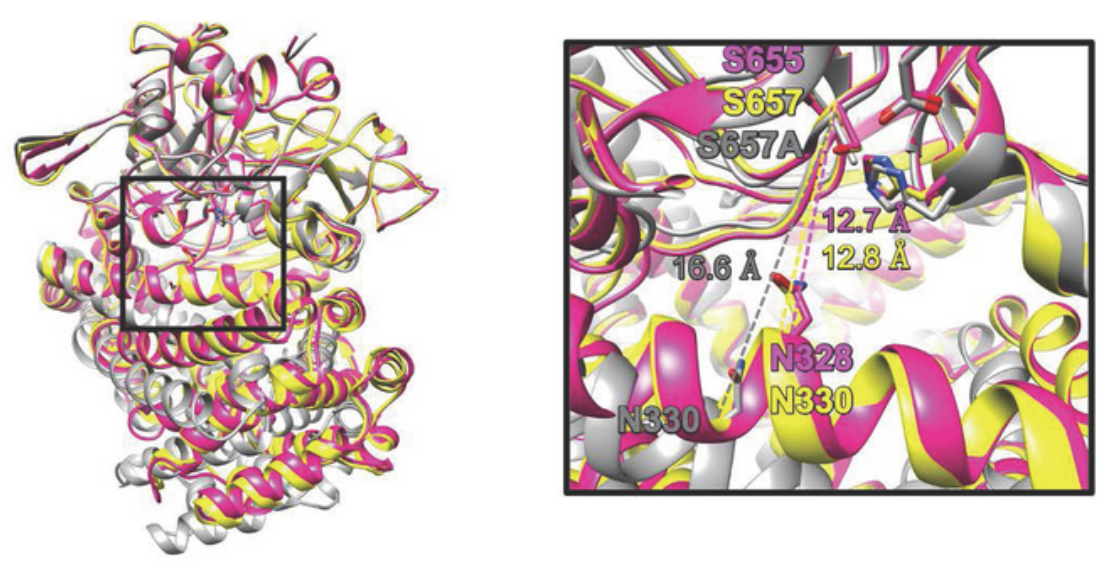

\section{Figure 4}

Superpositions of the protomers of SmDPP7, PmDAP BII, and PgDPP11 (a) Superposition of catalytic domains. Ribbon diagrams of SmDPP7 complexed with Tyr-Tyr dipeptide (PDB, 7DKC), PmDAP BII complexed with Val-Tyr dipeptide (PDB, 3WOL), and PgDPP11 complexed with citrate ion (PDB, 6JTB) are shown in magenta, yellow, and blue, respectively. Residues 23-274 and 572-720 of SmDPP7 are shown as a catalytic domain. Residues 25-276 and 574-720 of PmDAP BII and residues 22-279 and 572-720 
of PgDPP11, corresponding to the catalytic domain of SmDPP7, are shown. $\neg$ (b) The superposition of the protomers of SmDPP7 (PDB, 7DKC), PmDAP BII (PDB, 3WOL), and PgDPP11 (PDB, 6JTB). The colour coding is the same as (a). (c) The superposition of the protomers of SmDPP7 and PmDAPBII. Ribbon diagrams of SmDPP7 (PDB, 7DKC), PmDAP BII (PDB, 3WOL; closed conformation), and PmDAP BII (PDB, 3WOK; open conformation) are shown in magenta, yellow, and grey, respectively.
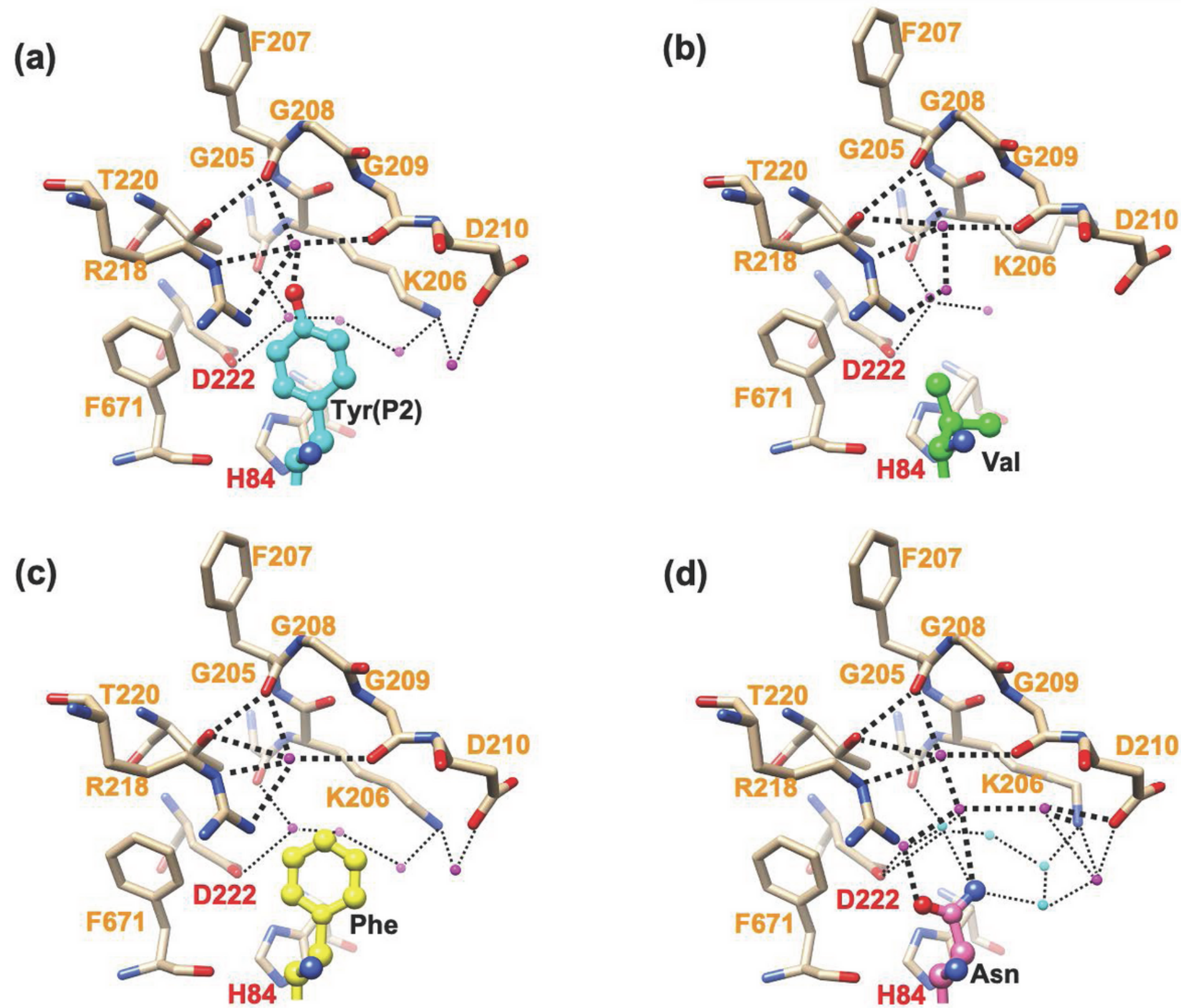

\section{Figure 5}

The S2 subsite of SmDPP7/dipeptide complexes Catalytic residues and S2 subsite residues are denoted in red and orange, respectively. Magenta and cyan spheroidal denote a water molecule. (a) Tyr-Tyr

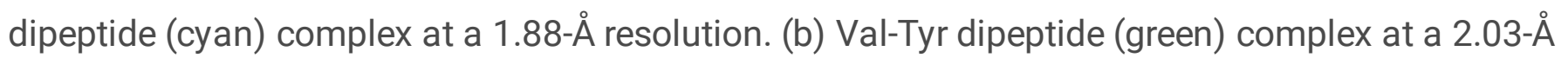
resolution. (c) Phe-Tyr dipeptide (yellow) complex at a 1.91-Å resolution. (d) Asn-Tyr dipeptide (pink) 
complex at a 1.92- $\AA$ resolution. Cyan spheroids denote a water molecule associated with a pentagonal hydrogen-bond network consisting of $\mathrm{HOH} 35, \mathrm{HOH} 63, \mathrm{HOH} 67, \mathrm{HOH} 1112$, and the ND2 atom of P2-Asn (see Figure S4).

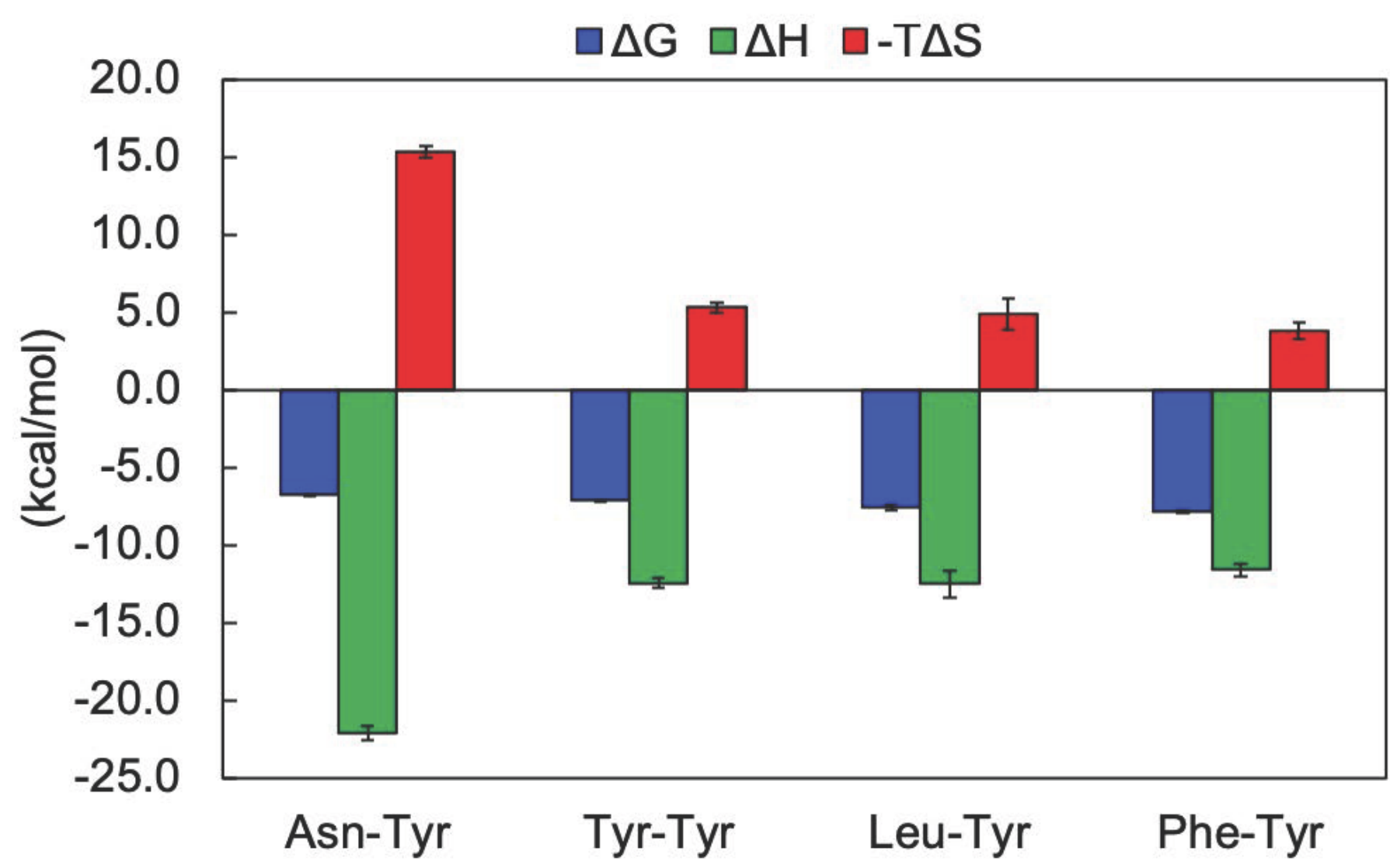

Figure 6

Thermodynamic parameters of dipeptide binding of SmDPP7 The calorimetric data for the respective dipeptide bindings are shown in Figure S5. The value of thermodynamic parameters is represented in Table S3. The dissociation constant $(\mathrm{Kd})$ and heats of binding $(\Delta \mathrm{H})$ were obtained using MICROCAL PEAQ-ITC Analysis software (Malvern, UK). Gibbs free energy $(\Delta G)$ and entropy energy $(\Delta S)$ were calculated according to the equation, $\Delta \mathrm{G}=-\mathrm{RT}$ In $\mathrm{Ka}=\mathrm{RT}$ In $\mathrm{Kd}$ ( $\mathrm{Ka}=1 / \mathrm{Kd}$, association constants). Standard deviations were obtained from three independent experiments.

\section{Supplementary Files}

This is a list of supplementary files associated with this preprint. Click to download.

- supplementaryinformation.pdf 